\title{
Search for the standard model Higgs boson in the $\mathrm{H} \rightarrow \mathrm{ZZ} \rightarrow 2 \ell 2 \nu$ channel in pp collisions at $\sqrt{s}=7 \mathrm{TeV}$
}

\section{The CMS collaboration}

ABStRaCT: A search for the standard model Higgs boson in the $\mathrm{H} \rightarrow \mathrm{ZZ} \rightarrow 2 \ell 2 \nu$ decay channel, where $\ell=\mathrm{e}$ or $\mu$, in pp collisions at a center-of-mass energy of $7 \mathrm{TeV}$ is presented. The data were collected at the LHC, with the CMS detector, and correspond to an integrated luminosity of $4.6 \mathrm{fb}^{-1}$. No significant excess is observed above the background expectation, and upper limits are set on the Higgs boson production cross section. The presence of the standard model Higgs boson with a mass in the $270-440 \mathrm{GeV}$ range is excluded at $95 \%$ confidence level.

KEYwORDS: Hadron-Hadron Scattering 


\section{Contents}

1 Introduction 1

2 CMS detector and simulations $\quad 2$

3 Event selection $\quad 2$

4 Analysis strategy 4

5 Background estimation 5

$\begin{array}{lll}6 & \text { Systematic uncertainties } & 7\end{array}$

$\begin{array}{lll}7 & \text { Results } & 8\end{array}$

$\begin{array}{lll}8 & \text { Summary } & 10\end{array}$

$\begin{array}{ll}\text { The CMS collaboration } & 15\end{array}$

\section{Introduction}

The standard model (SM) of particle physics [1-3] accommodates essentially all relevant experimental data. One of the remaining questions is the origin of mass for fundamental particles. Within the SM, vector boson masses arise from the spontaneous breaking of electroweak symmetry [4-9]. The existence of the associated field quantum, the Higgs boson, has yet to be experimentally established. The discovery or exclusion of the SM Higgs boson is one of the main goals of the physics programme at the CERN Large Hadron Collider (LHC).

To date, experimental searches for the SM Higgs boson have yielded null results. Limits at $95 \%$ confidence level $(\mathrm{CL})$ on its mass $\left(m_{\mathrm{H}}\right)$ have been placed by experiments at the Large Electron-Positron Collider (LEP), $m_{\mathrm{H}}>114.4 \mathrm{GeV}[10]$, the Tevatron, $m_{\mathrm{H}} \notin$ $(162-166) \mathrm{GeV}$ [11], and ATLAS, $m_{\mathrm{H}} \notin(145-206),(214-224)$, and $(340-450) \mathrm{GeV}$ [12-14]. The primary production mechanism for the Higgs boson at the LHC is through gluon fusion [15-26] with a small contribution from vector boson fusion (VBF) [27-29].

A search for the SM Higgs boson is presented in the $\mathrm{H} \rightarrow \mathrm{ZZ} \rightarrow 2 \ell 2 \nu$ channel (where $\ell$ refers to either e or $\mu$ ), which is especially sensitive in the high-mass range $250-600 \mathrm{GeV}$. Results are reported from a data sample corresponding to an integrated luminosity of $4.6 \mathrm{fb}^{-1}$ recorded in 2011 by the Compact Muon Solenoid (CMS) experiment at $\sqrt{s}=7 \mathrm{TeV}$. 


\section{CMS detector and simulations}

A detailed description of the CMS detector can be found in ref. [30]. The key components of the detector include a silicon pixel and a silicon strip tracker, embedded in a $3.8 \mathrm{~T}$ solenoidal magnetic field, used to measure the momentum of charged particles. The silicon pixel and strip tracking system covers the pseudorapidity range $|\eta|<2.5$, where $\eta=-\ln [\tan (\theta / 2)]$, and $\theta$ is the polar angle of the trajectory of the particle with respect to the beam direction. It is surrounded by a crystal electromagnetic calorimeter (ECAL) and a brass-scintillator hadron calorimeter (HCAL). The ECAL and HCAL extend to a pseudorapidity range of $|\eta|<3.0$. A steel/quartz-fiber Cherenkov forward detector (HF) extends the calorimetric coverage to $|\eta|<5.2$. The calorimeters are surrounded by the muon system, used to identify muons and measure their momentum. The muon system consists of gas detectors placed in the steel return yoke of the magnet.

The largest background to the SM Higgs boson signal consists of events in which a $\mathrm{Z}$ boson is produced in association with jets $(\mathrm{Z}+$ jets $)$. The $\mathrm{Z}+$ jets cross section is five orders of magnitude larger than the expected production cross section for the signal. The other major backgrounds are top-quark production ( $\mathrm{t} \overline{\mathrm{t}} \rightarrow 2 \ell 2 \nu 2 \mathrm{~b}$ and $\mathrm{tW} \rightarrow 2 \ell 2 \nu \mathrm{b}$, and the diboson production ( $\mathrm{WZ} \rightarrow 3 \ell \nu, \mathrm{ZZ} \rightarrow 2 \ell 2 \nu$, and $\mathrm{WW} \rightarrow 2 \ell 2 \nu$ ).

Several Monte Carlo event generators are used to simulate the signal and background processes. The $\mathrm{H} \rightarrow \mathrm{ZZ} \rightarrow 2 \ell 2 \nu$ signal and top-quark background events are generated by using the next-to-leading order (NLO) program POwHEG 2.0 [31]. The $\mathrm{Z}+$ jets and diboson backgrounds are simulated by using the MADGRAPH 5.1.3 generator [32]. The diboson backgrounds are also simulated using the PYTHIA 6.4.22 generator for evaluating certain systematic uncertainties. For events generated using POWHEG and MADGrapH generators, parton showering is simulated by using PYTHIA with the Z2 tune which differs from the Z1 tune described in ref. [33] as it uses the CTEQ6 [34] parametrization for the parton distribution functions instead of the CTEQ5 [35] parametrization. The signal events are reweighted so that the transverse momentum $\left(p_{\mathrm{T}}\right)$ distribution of the Higgs boson agrees with the next-to-next-to-leading order (NNLO) and next-to-next-to-leading $\log$ (NNLL) prediction [36, 37]. The total cross section is taken from ref. [38] and is scaled by the $\mathrm{H} \rightarrow \mathrm{ZZ} \rightarrow 2 \ell 2 \nu$ branching ratio [39-44]. The parton distribution functions (PDF) are modeled through the CTEQ6L [34] parametrization at leading order and the CT10 parametrization [45] at NLO. The NLO contribution to the qq $\rightarrow \mathrm{ZZ}$ process is taken into account by reweighting the transverse momentum of the visible $\mathrm{Z}$ boson to match the prediction from the MCFM 6.0 program [46]. A correction of $12 \%$ of the leading order $\mathrm{qq} \rightarrow \mathrm{ZZ}$ cross section is included to account for the $\mathrm{gg} \rightarrow \mathrm{ZZ}$ process [47]. The detector response to the simulated events is modeled with GEANT4 $[30,48]$ and reconstruction and analysis are performed by using the same software used for data.

\section{Event selection}

For Higgs boson masses considered in this analysis, the $\mathrm{Z}$ bosons from $\mathrm{H} \rightarrow \mathrm{ZZ}$ decay are typically produced with a substantial $p_{\mathrm{T}}$. Events are therefore selected to have two 
well-identified, isolated, opposite-charge leptons of same flavour $\left(\mathrm{e}^{+} \mathrm{e}^{-}\right.$or $\left.\mu^{+} \mu^{-}\right)$with $p_{\mathrm{T}}>$ $20 \mathrm{GeV}$ that have an invariant mass within $30 \mathrm{GeV}$ window centred on the $\mathrm{Z}$ mass. The $p_{\mathrm{T}}$ of the dilepton system is required to be greater than $55 \mathrm{GeV}$. In the electron channel, these events are collected by using dielectron triggers, with thresholds of $p_{\mathrm{T}}>17 \mathrm{GeV}$ and $p_{\mathrm{T}}>8 \mathrm{GeV}$ for the leading and the other electron, respectively. The muon channel relies on a combination of single- and double-muon triggers. As instantaneous luminosity increased, the thresholds on the double-muon triggers changed from a requirement of $p_{\mathrm{T}}>7 \mathrm{GeV}$ for each of the two muons to $p_{\mathrm{T}}>17 \mathrm{GeV}$ and $p_{\mathrm{T}}>8 \mathrm{GeV}$ on the leading and the other muon, respectively. The threshold for the single-muon trigger increased from $p_{\mathrm{T}}>17 \mathrm{GeV}$ to $p_{\mathrm{T}}>24 \mathrm{GeV}$. The trigger efficiency for signal, for events selected through the full set of offline requirements is measured by using $\mathrm{Z}$ decays in data, and ranges from $95 \%$ to $97 \%$ in the muon channel and exceeds $99 \%$ in the electron channel.

Muon candidates are reconstructed by using two algorithms, one in which tracks in the silicon tracker are matched to energy deposits in the muon detectors and another in which a combined fit is performed to signals in both the silicon tracker and the muon system [49]. The muon candidates for analysis are required to be successfully reconstructed through both algorithms. Other identification criteria based on the number of measurements in the tracker and in the muon system, the fit quality of the muon track, and its consistency with the origin from the primary vertex are also imposed on the muon candidates to reduce the misidentification rate.

Electron reconstruction also involves two algorithms [50], one in which energy clusters in the ECAL are matched to signals in the silicon tracker and another in which tracks in the silicon tracker are matched to ECAL clusters. The electron candidates used in the analysis can be reconstructed by either algorithm. More identification criteria based on the distribution of the shower in the ECAL, a matching of the trajectory of an electron track with the cluster in the ECAL, and consistency with origin of the track from the primary vertex are imposed on the electron candidates to reduce the misidentification rate. Electron candidates with an ECAL cluster in the transition region between ECAL barrel and endcap $(1.4442<|\eta|<1.566)$ are rejected. Additional requirements are imposed to remove electrons produced in photon conversions in the detector material.

Leptons produced in the decay of $\mathrm{Z}$ bosons are expected to be isolated from hadronic activity in the event. The sum of scalar transverse momentum depositions in the calorimeters and the transverse momenta of tracks in a cone of radius 0.3 in $\eta-\phi$ space around each lepton, where $\phi$ is the azimuthal angle, is corrected by the contribution from the lepton and the ratio of this corrected sum divided by the lepton $p_{\mathrm{T}}$ is required to be smaller than $15 \%$ (10\%) for muons (electrons). To correct for the contribution to the isolation sum from pile-up interactions (overlapping minimum-bias events from other concurrent protonproton collisions), a median energy density $(\rho)$ is determined event by event [51]. Then the pile-up contribution to the isolation sum is estimated as the product of $\rho$ and the area of the cone in which the isolation sum is computed, and it is subtracted from the isolation sum to make it largely insensitive to pile-up. The combined reconstruction, identification and isolation efficiency is measured in data by using $\mathrm{Z}$ decays and ranges between $90 \%$ and $97 \%$ for muons, and between $70 \%$ and $90 \%$ for electrons, depending on the $p_{\mathrm{T}}$ and $\eta$ of the leptons. 
The high instantaneous luminosity delivered by the LHC provides an average of about 10 pile-up interactions per bunch crossing, leading to events with several possible primary vertices. The vertex with largest value of $\sum p_{T}^{2}$ for the associated tracks is chosen to be the reference vertex. According to simulation, this requirement provides the correct assignment for the primary vertex in more than $99 \%$ of both signal and background events.

The presence of a large imbalance in transverse momentum in an event $\left(E_{\mathrm{T}}^{\text {miss }}\right)$ is a fundamental feature of the signal. The value of $E_{\mathrm{T}}^{\text {miss }}$ is the modulus of the $\vec{E}_{\mathrm{T}}^{\text {miss }}$ vector computed as the negative of the vector sum of the transverse momenta of all reconstructed objects identified through the particle-flow algorithm, which aims to reconstruct all particles produced in a collision event by combining information from all sub-detectors [52]. A large $E_{\mathrm{T}}^{\text {miss }}$ threshold is imposed to suppress the bulk of the $\mathrm{Z}+$ jets background, which contains little genuine $E_{\mathrm{T}}^{\text {miss }}$. The region of large $E_{\mathrm{T}}^{\text {miss }}$ is populated by $\mathrm{Z}+$ jets events in which the $E_{\mathrm{T}}^{\text {miss }}$ is largely due to jet mismeasurement. To suppress the background with $E_{\mathrm{T}}^{\text {miss }}$ arising from mismeasurement of jets, events are removed if the angle in the azimuthal plane between the $E_{\mathrm{T}}^{\text {miss }}$ and the closest jet with transverse energy $E_{\mathrm{T}}>30 \mathrm{GeV}$ is smaller than 0.5 radians. For events having no jets with $E_{\mathrm{T}}>30 \mathrm{GeV}$, this requirement is imposed between $E_{\mathrm{T}}^{\text {miss }}$ and the closest jet with $E_{\mathrm{T}}>15 \mathrm{GeV}$. Jets are reconstructed from particle-flow candidates $[52,53]$ by using the anti- $\mathrm{k}_{\mathrm{T}}$ clustering algorithm [54] with a distance parameter $\mathrm{R}$ of 0.5 , as implemented in the FASTJET package $[55,56]$.

Top-quark decays are characterized by the presence of jets originating from $b$ quarks (bjets), which are tagged on the basis of impact parameters of tracks in a jet, relative to the primary vertex $[57,58]$. The top-quark background is suppressed by applying a veto on events having a $\mathrm{b}$ tagged jet with transverse energy greater than $30 \mathrm{GeV}$ that lies within the tracker volume $(|\eta|<2.4)$. To further suppress the top-quark background, a veto is applied on events containing a "soft muon" with $p_{\mathrm{T}}>3 \mathrm{GeV}$, which is typically produced in the leptonic decay of a $b$ quark. The soft-muon veto along with the b-jet veto reduces the top-quark background by a factor of six. To reduce the WZ background in which both bosons decay leptonically, any event with a third lepton (e or $\mu$ ) with $p_{\mathrm{T}}>10 \mathrm{GeV}$ and passing the identification and isolation requirements is rejected.

\section{Analysis strategy}

The search for the SM Higgs boson is performed by using a transverse mass $\left(M_{\mathrm{T}}\right)$ variable as the final discriminant in searching for an excess of events from the presence of the signal. The transverse mass is defined as follows:

$$
M_{\mathrm{T}}^{2}=\left(\sqrt{p_{\mathrm{T}}(\ell \ell)^{2}+M(\ell \ell)^{2}}+\sqrt{E_{\mathrm{T}}^{\mathrm{miss}}{ }^{2}+M(\ell \ell)^{2}}\right)^{2}-\left(\vec{p}_{T}(\ell \ell)+\vec{E}_{T}^{\mathrm{miss}}\right)^{2} .
$$

where $p_{\mathrm{T}}(\ell \ell)$ and $M(\ell \ell)$ are the transverse momentum and invariant mass of dilepton system, respectively.

Two approaches are considered: a "cut-based" and a "shape-based" analysis. The same set of event selection criteria is used by both the analyses, except for requirements on the $E_{\mathrm{T}}^{\mathrm{miss}}$ and $M_{\mathrm{T}}$ variables. In both cases, requirements on $E_{\mathrm{T}}^{\mathrm{miss}}$ and $M_{\mathrm{T}}$ vary with the Higgs mass hypothesis, but are typically more relaxed for the shape-based analysis. 


\begin{tabular}{|c|cccccc|}
\hline$m_{\mathrm{H}}(\mathrm{GeV})$ & 250 & 300 & 350 & 400 & 500 & 600 \\
\hline \multicolumn{7}{|c|}{ Cut-based analysis selection } \\
\hline$E_{\mathrm{T}}^{\text {miss }}(\mathrm{GeV})$ & $>70$ & $>79$ & $>95$ & $>115$ & $>150$ & $>161$ \\
$M_{T}(\mathrm{GeV})$ & {$[222,272]$} & {$[264,331]$} & {$[298,393]$} & {$[327,460]$} & {$[382,605]$} & {$[452,767]$} \\
\hline \multicolumn{7}{|c|}{ Shape-based analysis selection } \\
\hline$E_{\mathrm{T}}^{\text {miss }}(\mathrm{GeV})$ & $>70$ & $>80$ & $>80$ & $>80$ & $>80$ & $>80$ \\
$M_{T}(\mathrm{GeV})$ & {$[180,300]$} & {$[250,350]$} & {$[250,400]$} & {$[250,450]$} & {$[250,600]$} & {$[250,750]$} \\
\hline
\end{tabular}

Table 1. Higgs boson mass-dependent selection for $E_{\mathrm{T}}^{\mathrm{miss}}$ and $M_{\mathrm{T}}$ variables in the cut-based and shape-based analyses.

In the cut-based analysis, the Higgs boson search is performed by looking for an excess of events above the standard model background expectation after applying selection criteria that are tuned for a given Higgs boson mass hypothesis. The $E_{\mathrm{T}}^{\mathrm{miss}}$ and $M_{\mathrm{T}}$ selection listed in table 1 was optimized by using a genetic algorithm for rectangular cuts optimization, GARCON [59], with the expected 95\% CL limit on the cross section normalized to the standard model Higgs boson cross section as a figure of merit. Optimization was performed on six $m_{\mathrm{H}}$ points, and a smooth-fit interpolation for the cut values was performed to provide optimal cut values for every explored Higgs mass point.

In the shape-based analysis, the Higgs boson search is performed by using a binned likelihood fit to the $M_{\mathrm{T}}$ distribution obtained after applying the selection given in table 1 . The range of $M_{\mathrm{T}}$ is varied as a function of $m_{\mathrm{H}}$ to ensure that the expected signal distribution is fully contained. The shape-based analysis is found to have the best expected signal sensitivity and is considered as the main result in the paper.

\section{$5 \quad$ Background estimation}

For both types of analyses, the ZZ and WZ backgrounds are modeled using Monte Carlo simulation, and are normalized to their respective NLO cross sections. The remaining backgrounds are estimated using control samples in data.

The $\mathrm{Z}+$ jets background is simulated from a control sample of events with a single photon produced in association with jets $(\gamma+$ jets $)$. This obviates the need for using less reliable Monte Carlo simulation of the $E_{\mathrm{T}}^{\text {miss }}$ distribution arising from mismeasurement of jets. The $\gamma+$ jets cross section is much larger than $\mathrm{Z}+$ jets. The jets must have a minimum $E_{\mathrm{T}}$ of $15 \mathrm{GeV}$ to reduce contamination from processes that have a photon produced in association with genuine $E_{\mathrm{T}}^{\text {miss }}$, such as $\mathrm{W}(\ell \nu)+\gamma, \mathrm{W}(\ell \nu)+$ jets, where the jet is mismeasured as a photon, and $\mathrm{Z}(\nu \nu)+\gamma$ events. The kinematics and overall normalization of $\gamma+$ jets events are matched to $\mathrm{Z}+$ jets in data through an event-by-event reweighting as a function of the boson $p_{\mathrm{T}}$, and the number of jets with $E_{\mathrm{T}}>30 \mathrm{GeV}$, to account for the dependence of the $E_{\mathrm{T}}^{\mathrm{miss}}$ on the associated hadronic activity. Residual differences from pileup are taken into account by reweighting events according to the number of reconstructed vertices. This procedure yields an accurate model of the $E_{\mathrm{T}}^{\text {miss }}$ distribution in $\mathrm{Z}+$ jets events, 


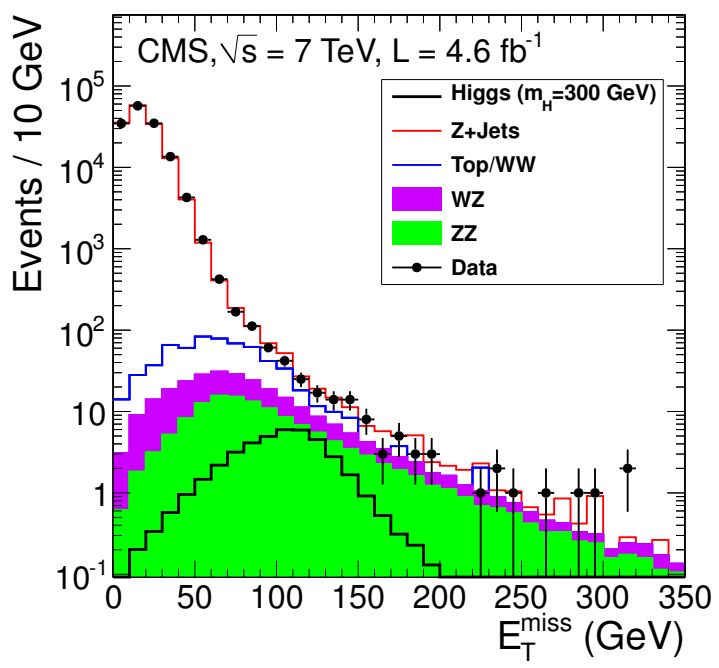

Figure 1. The $E_{\mathrm{T}}^{\text {miss }}$ distribution in data compared to the background simulation. The dielectron and dimuon channels are combined. Contributions from ZZ, WZ, non-resonant background and $\mathrm{Z}+$ jets background are stacked on top of each other. The $E_{\mathrm{T}}^{\mathrm{miss}}$ distribution in signal events for $m_{\mathrm{H}}=300 \mathrm{GeV}$ is also shown.

as shown in figure 1, which compares the $E_{\mathrm{T}}^{\text {miss }}$ distribution of the reweighted $\gamma+$ jets events along with other backgrounds to the $E_{\mathrm{T}}^{\mathrm{miss}}$ distribution of the dilepton events in data. To compute the $M_{\mathrm{T}}$ for each $\gamma+$ jets event, the value of $\vec{p}_{\mathrm{T}}(\ell \ell)$ is defined as the photon $\vec{p}_{\mathrm{T}}$ and the value of $M(\ell \ell)$ term is chosen according to a probability density function constructed from the measured dilepton invariant mass distribution in $\mathrm{Z}+$ jets events.

The background processes that do not involve a $\mathrm{Z}$ resonance (non-resonant background) are estimated by using a control sample of events with dileptons of different flavor $\left(\mathrm{e}^{ \pm} \mu^{\mp}\right)$ that pass the full analysis selection. This background consists mainly of leptonic W decays in $\mathrm{t} \overline{\mathrm{t}}, \mathrm{tW}$ decays and $\mathrm{WW}$ events. Small contributions from single top-quark events produced from $s$-channel and $t$-channel processes, $\mathrm{W}+$ jets events in which the $\mathrm{W}$ boson decays leptonically and a jet is mismeasured as a lepton, and $\mathrm{Z} \rightarrow \tau \tau$ events in which $\tau$ leptons produce light leptons and $E_{\mathrm{T}}^{\text {miss }}$ are included in this estimate of the non-resonant background. This method cannot distinguish between the non-resonant background and a possible contribution from $\mathrm{H} \rightarrow \mathrm{WW} \rightarrow 2 \ell 2 \nu$ events, which are treated as part of the non-resonant background estimate.

The non-resonant background in the $\mathrm{e}^{+} \mathrm{e}^{-}$and $\mu^{+} \mu^{-}$final states is estimated by applying a scale factor $(\alpha)$ to the selected $\mathrm{e}^{ \pm} \mu^{\mp}$ events:

$$
N_{\mu \mu}=\alpha_{\mu} \times N_{\mathrm{e} \mu}, \quad N_{\mathrm{ee}}=\alpha_{\mathrm{e}} \times N_{\mathrm{e} \mu} .
$$

This $\alpha$ factor is computed from the sidebands (SB) of the $\mathrm{Z}$ peak $(40<M(\ell \ell)<$ $70 \mathrm{GeV}$ and $110<M(\ell \ell)<200 \mathrm{GeV})$ by using the following relations:

$$
\alpha_{\mu}=\frac{N_{\mu \mu}^{\mathrm{SB}}}{N_{\mathrm{e} \mu}^{\mathrm{SB}}}, \quad \alpha_{\mathrm{e}}=\frac{N_{\mathrm{ee}}^{\mathrm{SB}}}{N_{\mathrm{e} \mu}^{S B}},
$$


where $N_{\mathrm{ee}}^{\mathrm{SB}}, N_{\mu \mu}^{\mathrm{SB}}$, and $N_{\mathrm{e} \mu}^{\mathrm{SB}}$ are the number of events in the $\mathrm{Z}$ sidebands in a top-enriched sample of $\mathrm{e}^{+} \mathrm{e}^{-}, \mu^{+} \mu^{-}$, and $\mathrm{e}^{ \pm} \mu^{\mp}$ final states, respectively. Such samples are selected by requiring $E_{\mathrm{T}}^{\mathrm{miss}}>70 \mathrm{GeV}$ and a b-tagged jet in the events. The measured values of $\alpha$ with the corresponding statistical uncertainties are $\alpha_{\mu}=0.58 \pm 0.02$ and $\alpha_{\mathrm{e}}=0.42 \pm 0.02$.

Since the $M_{\mathrm{T}}$ distribution obtained for the non-resonant background is based on a small sample of $\mathrm{e}^{ \pm} \mu^{\mp}$ events scaled by $\alpha$, this distribution is smoothed [60] for use in the shape-based analysis.

\section{$6 \quad$ Systematic uncertainties}

Systematic uncertainties include experimental uncertainties on the selection and measurement of the reconstructed objects, theoretical uncertainties on the signal and background processes which are derived from Monte Carlo simulation, and uncertainties on backgrounds determined from control samples in data. These are summarised in table 2.

The theoretical uncertainties on the predicted signal are evaluated by changing the parton distribution functions and the QCD renormalization and factorization scales [38, 45, 61-64]. The theoretical uncertainties on the NLO cross sections of the ZZ and WZ backgrounds are estimated by the same procedure of changing the parton distribution functions and the QCD renormalization and factorization scales by using the program MCFM 6.0.

The cross section for on-shell Higgs boson production and decay is calculated in the zero-width approximation. Recent studies show that current Monte Carlo simulation does not describe the correct Higgs-boson line shape for $m_{\mathrm{H}} \gtrsim 300 \mathrm{GeV}$. These effects are estimated to correspond to an additional uncertainty of $10-30 \%$ on the theoretical cross section for $400<m_{\mathrm{H}}<600 \mathrm{GeV}[38,65,66]$.

Various factors contribute to the experimental uncertainties that apply to processes derived from Monte Carlo simulation. These include uncertainties on the trigger efficiency and lepton selection efficiencies. The effect of lepton momentum scale and jet energy scale is also taken into account and is propagated to the evaluation of $E_{\mathrm{T}}^{\mathrm{miss}}$. The uncertainty on the $b$ jet veto is estimated by measuring the b-tagging efficiency in data from fully leptonic $t \bar{t}$ decays [58]. The uncertainty due to the modeling of pile-up is evaluated by shiftng the mean of the distribution of the number of pile-up interactions up and down by one.

The uncertainty on the estimate of the non-resonant background is dominated by the statistical uncertainty of the $\mathrm{e}^{ \pm} \mu^{\mp}$ control sample. The uncertainty on the prediction of the $\mathrm{Z}+$ jets background is affected by any residual contamination of the $\gamma+$ jets control sample from processes involving a photon and genuine $E_{\mathrm{T}}^{\mathrm{miss}}$. Consequently, this method provides only an upper bound on the prediction. To account for this contamination, the $\mathrm{Z}+$ jets background prediction is changed from zero to the estimate obtained from the $\gamma+$ jets control sample in the limit-setting procedure. This is achieved by taking half of the estimate from the $\gamma+$ jets control sample as the central value for the $\mathrm{Z}+$ jets background and assigning a $100 \%$ systematic uncertainty to this value. Since $\mathrm{Z}+$ jets contributes to only $10-15 \%$ of the total background, the large uncertainty on this background does not have a significant impact on the overall performance of the analysis. 


\begin{tabular}{|l|l|}
\hline Source & Uncertainty [\%] \\
\hline \hline Luminosity & 4.5 \\
\hline PDF for Higgs production (gluon-fusion) & $8.2-10.4$ \\
\hline PDF for Higgs production (VBF) & $3.8-7.6$ \\
\hline PDF for qq $\rightarrow$ ZZ & 4.8 \\
\hline PDF for qq $\rightarrow$ WZ & 5.5 \\
\hline QCD scale for Higgs production (gluon-fusion) & $7.6-11.1$ \\
\hline QCD scale for Higgs production (VBF) & $0.4-2$ \\
\hline QCD scale for gg $\rightarrow$ ZZ & 20 \\
\hline QCD scale for qq $\rightarrow$ ZZ & 6.2 \\
\hline QCD scale for qq $\rightarrow$ WZ & 8.5 \\
\hline Higgs boson line shape & $10-30$ \\
\hline Trigger & $1($ for ee), $2($ for $\mu \mu)$ \\
\hline Lepton identification and isolation & 2 \\
\hline Lepton momentum scale & $5($ for ee), $2($ for $\mu \mu)$ \\
\hline Jet energy scale & $1-1.5$ \\
\hline b-jet veto & $1-1.2$ \\
\hline Pile-up & $1-3$ \\
\hline Non-resonant background & $15-100$ \\
\hline Z+jets & 100 \\
\hline
\end{tabular}

Table 2. Summary of systematic uncertainties on event yields of signal and background processes. Ranges refer to uncertainties that depend on the hypothesised value of $m_{\mathrm{H}}$.

In the shape-based analysis, the difference in the $M_{\mathrm{T}}$ distribution with respect to an alternate Monte Carlo generator (MADGRAPH versus PYTHIA) is used as an uncertainty in the modeling of the ZZ, WZ backgrounds. The uncertainty on the $M_{\mathrm{T}}$ distributions arising from the limited number of Monte Carlo simulated events is taken into account by scaling all the bins up and down by the respective statistical uncertainties. This is done in a correlated manner to obtain two bounding distributions, within which the nominal distribution is changed. For the non-resonant background, where the nominal $M_{\mathrm{T}}$ distribution is obtained by smoothing the $M_{\mathrm{T}}$ distribution of $\mathrm{e}^{ \pm} \mu^{\mp}$ events, the bounding distributions are taken from simulation to account for any possible bias introduced by smoothing. The uncertainty for signal is assigned by reweighting the $p_{\mathrm{T}}$ spectrum of the Higgs boson to the one obtained from changing the renormalization and factorization scales in the NNLO+NNLL calculation [36, 37].

\section{$7 \quad$ Results}

The event yields for the cut-based analysis, as a function of $m_{\mathrm{H}}$, and the estimated backgrounds for the $4.6 \mathrm{fb}^{-1}$ dataset are listed in table 3 . The $M_{\mathrm{T}}$ distributions for the shapebased analysis based on the selections described in table 1 for $m_{\mathrm{H}}=300$ and $400 \mathrm{GeV}$ hypotheses are shown in figure 2. No significant excess of events is observed over the ex- 


\begin{tabular}{|l|c|c|c|c|c|c|c|}
\hline $\begin{array}{l}m_{\mathrm{H}} \\
(\mathrm{GeV})\end{array}$ & ZZ & WZ & $\begin{array}{c}\text { Top/WW/ } \\
\text { W+jets/Z } \rightarrow \tau \tau\end{array}$ & Z+Jets & $\begin{array}{c}\text { Total } \\
\text { Background }\end{array}$ & $\begin{array}{c}\text { Expected } \\
\text { Signal }\end{array}$ & Data \\
\hline \hline 250 & $36.0 \pm 0.2 \pm 2.6$ & $24.0 \pm 0.3 \pm 2.0$ & $65.0 \pm 3.8 \pm 5.8$ & $15.0 \pm 15.0$ & $140.0 \pm 3.8 \pm 16.0$ & $22.0 \pm 2.2$ & 142 \\
\hline 300 & $23.0 \pm 0.2 \pm 1.7$ & $13.0 \pm 0.2 \pm 1.1$ & $18.0 \pm 1.1 \pm 3.0$ & $6.3 \pm 6.3$ & $60.0 \pm 1.1 \pm 7.3$ & $21.0 \pm 2.1$ & 64 \\
\hline 350 & $16.0 \pm 0.1 \pm 1.1$ & $7.0 \pm 0.2 \pm 0.6$ & $2.0 \pm 0.1 \pm 1.0$ & $4.1 \pm 4.1$ & $29.0 \pm 0.3 \pm 4.4$ & $21.0 \pm 2.5$ & 26 \\
\hline 400 & $12.0 \pm 0.1 \pm 0.9$ & $4.6 \pm 0.1 \pm 0.4$ & $<1.1$ & $2.7 \pm 2.7$ & $19.0 \pm 0.2 \pm 2.9$ & $17.0 \pm 2.0$ & 18 \\
\hline 500 & $7.5 \pm 0.1 \pm 0.5$ & $2.0 \pm 0.1 \pm 0.2$ & $<1.1$ & $1.4 \pm 1.4$ & $11.0 \pm 0.1 \pm 1.5$ & $7.4 \pm 1.3$ & 14 \\
\hline 600 & $3.9 \pm 0.1 \pm 0.3$ & $0.8 \pm 0.1 \pm 0.1$ & $<1.1$ & $0.6 \pm 0.6$ & $5.3 \pm 0.1 \pm 0.7$ & $2.9 \pm 0.7$ & 5 \\
\hline
\end{tabular}

Table 3. Background estimates, signal predictions, and observed number of events for an integrated luminosity of $4.6 \mathrm{fb}^{-1}$ after the cut-based selection. The uncertainties include the statistical and systematic components (in that order). The results for signal contain only the systematic uncertainties as the statistical uncertainties are negligible. For the non-resonant background, the $68 \%$ upper limit is quoted in the case where the background prediction is zero.
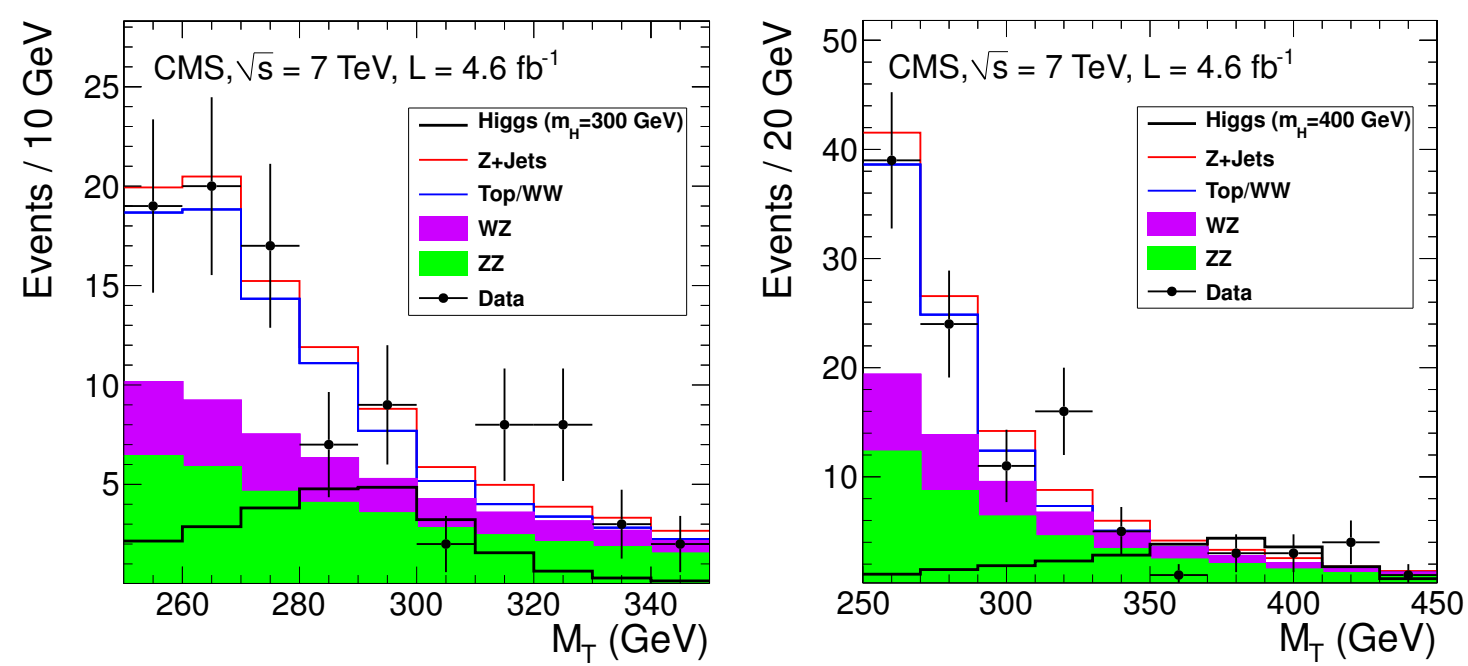

Figure 2. The $M_{\mathrm{T}}$ distribution for events passing $m_{\mathrm{H}}$ selections for $300 \mathrm{GeV}$ (left) and $400 \mathrm{GeV}$ (right). The dielectron and dimuon channels are combined.

pectation from the SM background and limits are set on the production cross section of the standard model Higgs boson as a function of $m_{\mathrm{H}}$.

The median expected and observed 95\% CL upper limits on the cross section $\sigma \times$ $B R(\mathrm{H} \rightarrow \mathrm{ZZ} \rightarrow 2 \ell 2 \nu)$ for $250<m_{\mathrm{H}}<600 \mathrm{GeV}$ by using the cut- and the shape-based approaches are shown in figure 3. The measured ratio $R$ of the $95 \%$ CL upper limit cross section $\sigma$ to the SM Higgs boson production cross section $\sigma_{\mathrm{SM}}$ as a function of $m_{\mathrm{H}}$ is shown in figure 4 . These results are obtained by using the $\mathrm{CL}_{\mathrm{s}}$ method [67-69]. For the cut-based analysis the SM Higgs boson is excluded in the mass range $310-465 \mathrm{GeV}$ at $95 \%$ confidence level, while the expected exclusion limit in the background-only hypothesis is $305-470 \mathrm{GeV}$. For the shape-based analysis the SM Higgs boson is excluded in the mass range $270-440 \mathrm{GeV}$ at $95 \%$ confidence level while the expected exclusion limit for the background-only hypothesis is $290-490 \mathrm{GeV}$. 

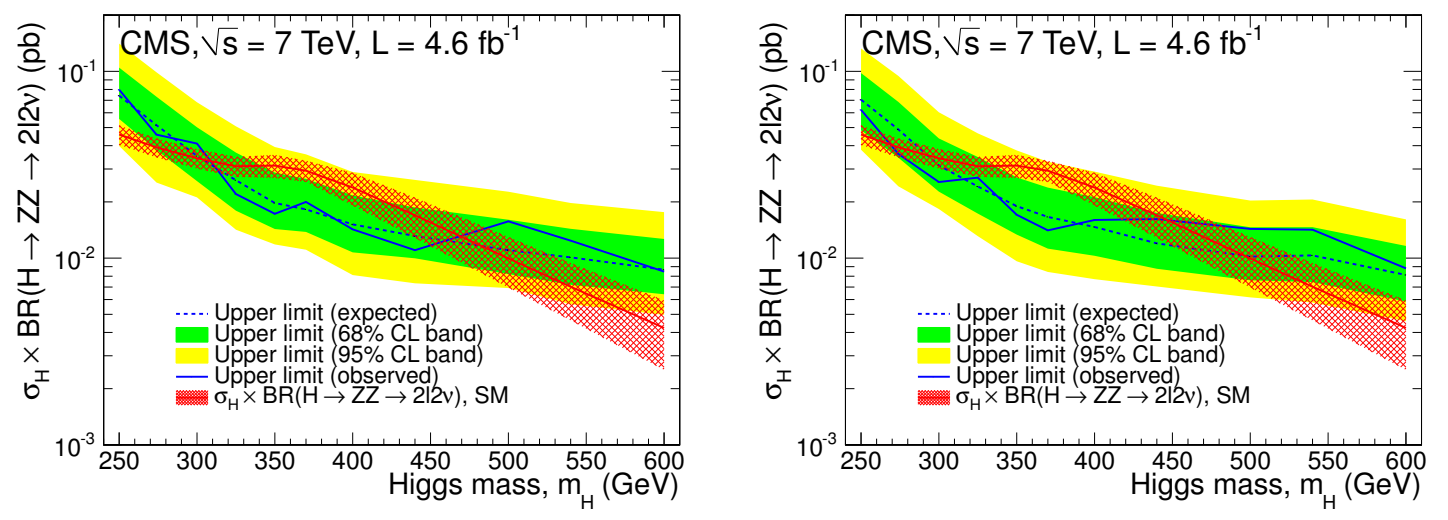

Figure 3. The median expected and observed 95\% CL upper limits on the cross section $\sigma \times$ $B R(H \rightarrow \mathrm{ZZ} \rightarrow 2 \ell 2 \nu)$ for the Higgs boson masses in the range $250-600 \mathrm{GeV}$ for the cut-based (left) and shape-based (right) analyses.
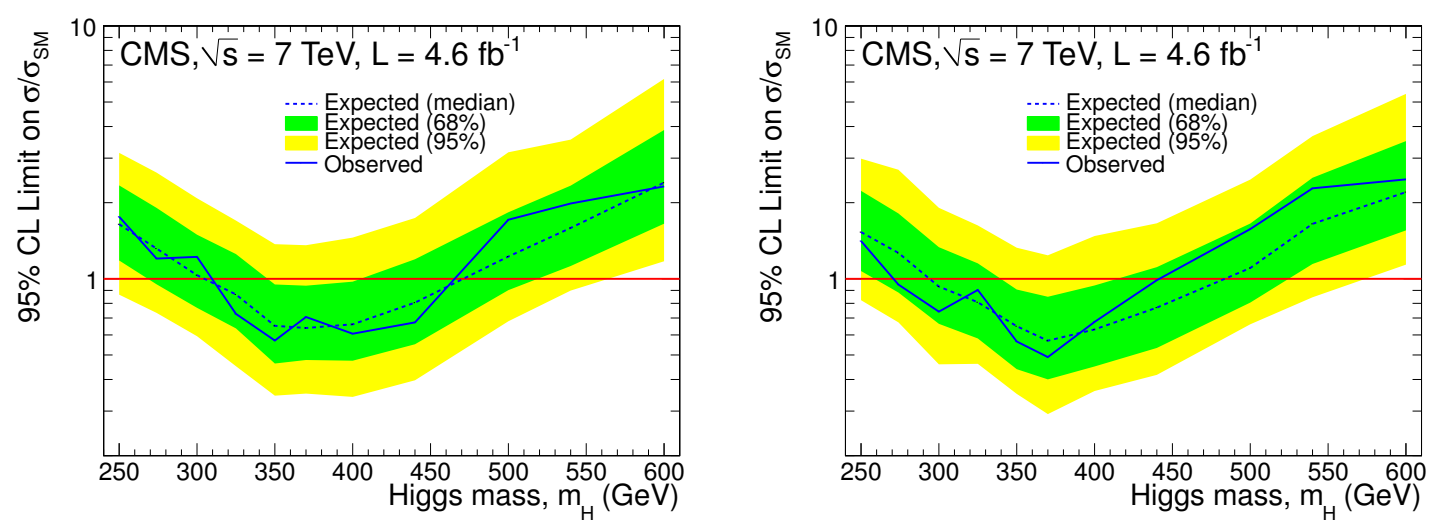

Figure 4. The ratio $R$ of the $95 \%$ CL cross section upper limit $\sigma$ to the SM Higgs boson production cross section $\sigma_{\mathrm{SM}}$ as a function of the Higgs boson mass $m_{\mathrm{H}}$ for the cut-based (left) and shape-based (right) analyses.

\section{Summary}

A search for the standard model Higgs boson has been performed in the decay channel $\mathrm{H} \rightarrow \mathrm{ZZ} \rightarrow 2 \ell 2 \nu$ in pp collisions at $\sqrt{s}=7 \mathrm{TeV}$, using a data sample corresponding to an integrated luminosity of $4.6 \mathrm{fb}^{-1}$. No significant excess is found above the background expectation. The presence of the SM Higgs boson is excluded for $270 \mathrm{GeV}<m_{\mathrm{H}}<440 \mathrm{GeV}$ at $95 \%$ CL.

\section{Acknowledgments}

We congratulate our colleagues in the CERN accelerator departments for the excellent performance of the LHC machine. We thank the technical and administrative staff at CERN and other CMS institutes, and acknowledge support from: FMSR (Austria); FNRS 
and FWO (Belgium); CNPq, CAPES, FAPERJ, and FAPESP (Brazil); MES (Bulgaria); CERN; CAS, MoST, and NSFC (China); COLCIENCIAS (Colombia); MSES (Croatia); RPF (Cyprus); MoER, SF0690030s09 and ERDF (Estonia); Academy of Finland, MEC, and HIP (Finland); CEA and CNRS/IN2P3 (France); BMBF, DFG, and HGF (Germany); GSRT (Greece); OTKA and NKTH (Hungary); DAE and DST (India); IPM (Iran); SFI (Ireland); INFN (Italy); NRF and WCU (Korea); LAS (Lithuania); CINVESTAV, CONACYT, SEP, and UASLP-FAI (Mexico); MSI (New Zealand); PAEC (Pakistan); MSHE and NSC (Poland); FCT (Portugal); JINR (Armenia, Belarus, Georgia, Ukraine, Uzbekistan); MON, RosAtom, RAS and RFBR (Russia); MSTD (Serbia); MICINN and CPAN (Spain); Swiss Funding Agencies (Switzerland); NSC (Taipei); TUBITAK and TAEK (Turkey); STFC (United Kingdom); DOE and NSF (U.S.A.). Individuals have received support from the Marie-Curie programme and the European Research Council (European Union); the Leventis Foundation; the A. P. Sloan Foundation; the Alexander von Humboldt Foundation; the Belgian Federal Science Policy Office; the Fonds pour la Formation à la Recherche dans l'Industrie et dans l'Agriculture (FRIA-Belgium); the Agentschap voor Innovatie door Wetenschap en Technologie (IWT-Belgium); the Council of Science and Industrial Research, India; and the HOMING PLUS programme of Foundation for Polish Science, cofinanced from European Union, Regional Development Fund.

Open Access. This article is distributed under the terms of the Creative Commons Attribution License which permits any use, distribution and reproduction in any medium, provided the original author(s) and source are credited.

\section{References}

[1] S. Glashow, Partial symmetries of weak interactions, Nucl. Phys. 22 (1961) 579.

[2] S. Weinberg, A model of leptons, Phys. Rev. Lett. 19 (1967) 1264 [InSPIRE].

[3] A. Salam, Weak and electromagnetic interactions, in Elementary particle physics: relativistic groups and analyticity. Proceedings of the eighth Nobel symposium, Lerum Sweden (1968), N. Svartholm, eds., Almquvist \& Wiskell, Stockholm Sweden (1968), pg. 367.

[4] F. Englert and R. Brout, Broken symmetry and the mass of gauge vector mesons, Phys. Rev. Lett. 13 (1964) 321 [INSPIRE].

[5] P.W. Higgs, Broken symmetries, massless particles and gauge fields, Phys. Lett. 12 (1964) 132 [INSPIRE].

[6] P.W. Higgs, Broken symmetries and the masses of gauge bosons, Phys. Rev. Lett. 13 (1964) 508 [INSPIRE].

[7] G. Guralnik, C. Hagen and T. Kibble, Global conservation laws and massless particles, Phys. Rev. Lett. 13 (1964) 585 [INSPIRE].

[8] P.W. Higgs, Spontaneous symmetry breakdown without massless bosons, Phys. Rev. 145 (1966) 1156 [INSPIRE].

[9] T. Kibble, Symmetry breaking in nonAbelian gauge theories, Phys. Rev. 155 (1967) 1554 [INSPIRE]. 
[10] ALEPH, DELPHI, L3, OPAL collaborations and LEP Working Group For HigGs Boson Searches, G. Abbiendi et al., Search for the Standard Model Higgs boson at LEP, Phys. Lett. B 565 (2003) 61 [hep-ex/0306033] [INSPIRE].

[11] CDF, D0 collaborations, T. Aaltonen et al., Combination of Tevatron searches for the standard model Higgs boson in the $W^{+} W^{-}$decay mode, Phys. Rev. Lett. 104 (2010) 061802 [arXiv:1001.4162] [INSPIRE]. A more recent, unpublished, limit is given in preprint arXiv:1103.3233.

[12] ATLAS collaboration, G. Aad et al., Search for the Standard Model Higgs boson in the decay channel $H \rightarrow Z Z^{(*)} \rightarrow 4 \ell$ with the ATLAS detector, Phys. Lett. B 705 (2011) 435 [arXiv: 1109.5945] [INSPIRE].

[13] ATLAS collaboration, Search for a Standard Model Higgs boson in the $H \rightarrow Z Z \rightarrow \ell^{+} \ell^{-} \nu \bar{\nu}$ decay channel with the ATLAS detector, Phys. Rev. Lett. 107 (2011) 221802 [arXiv:1109.3357] [INSPIRE].

[14] ATLAS collaboration, G. Aad et al., Search for the Higgs boson in the $H \rightarrow W W\left({ }^{*}\right) \rightarrow \ell^{+} \nu \ell^{-} \bar{\nu}$ decay channel in pp collisions at $\sqrt{s}=7 \mathrm{TeV}$ with the ATLAS detector (2011), submitted to Phys. Rev. Lett., arXiv:1112.2577 [INSPIRE].

[15] A. Djouadi, M. Spira and P. Zerwas, Production of Higgs bosons in proton colliders: QCD corrections, Phys. Lett. B 264 (1991) 440 [INSPIRE].

[16] S. Dawson, Radiative corrections to Higgs boson production, Nucl. Phys. B 359 (1991) 283 [INSPIRE].

[17] M. Spira, A. Djouadi, D. Graudenz and P. Zerwas, Higgs boson production at the LHC, Nucl. Phys. B 453 (1995) 17 [hep-ph/9504378] [INSPIRE].

[18] R.V. Harlander and W.B. Kilgore, Next-to-next-to-leading order Higgs production at hadron colliders, Phys. Rev. Lett. 88 (2002) 201801 [hep-ph/0201206] [INSPIRE].

[19] C. Anastasiou and K. Melnikov, Higgs boson production at hadron colliders in NNLO QCD, Nucl. Phys. B 646 (2002) 220 [hep-ph/0207004] [INSPIRE].

[20] V. Ravindran, J. Smith and W.L. van Neerven, NNLO corrections to the total cross-section for Higgs boson production in hadron hadron collisions, Nucl. Phys. B 665 (2003) 325 [hep-ph/0302135] [INSPIRE].

[21] U. Aglietti, R. Bonciani, G. Degrassi and A. Vicini, Two-loop light fermion contribution to Higgs production and decays, Phys. Lett. B 595 (2004) 432 [hep-ph/0404071] [INSPIRE].

[22] G. Degrassi and F. Maltoni, Two-loop electroweak corrections to Higgs production at hadron colliders, Phys. Lett. B 600 (2004) 255 [hep-ph/0407249] [INSPIRE].

[23] S. Actis, G. Passarino, C. Sturm and S. Uccirati, NLO Electroweak Corrections to Higgs Boson Production at Hadron Colliders, Phys. Lett. B 670 (2008) 12 [arXiv:0809.1301] [INSPIRE].

[24] C. Anastasiou, R. Boughezal and F. Petriello, Mixed QCD-electroweak corrections to Higgs boson production in gluon fusion, JHEP 04 (2009) 003 [arXiv:0811.3458] [INSPIRE].

[25] D. de Florian and M. Grazzini, Higgs production through gluon fusion: Updated cross sections at the Tevatron and the LHC, Phys. Lett. B 674 (2009) 291 [arXiv:0901.2427] [INSPIRE].

[26] J. Baglio and A. Djouadi, Higgs production at the LHC, JHEP 03 (2011) 055 [arXiv: 1012.0530] [INSPIRE]. 
[27] M. Ciccolini, A. Denner and S. Dittmaier, Strong and electroweak corrections to the production of Higgs + 2jets via weak interactions at the LHC, Phys. Rev. Lett. 99 (2007) 161803 [arXiv:0707.0381] [INSPIRE].

[28] M. Ciccolini, A. Denner and S. Dittmaier, Electroweak and QCD corrections to Higgs production via vector-boson fusion at the LHC, Phys. Rev. D 77 (2008) 013002 [arXiv:0710.4749] [INSPIRE].

[29] K. Arnold et al., VBFNLO: A parton level Monte Carlo for processes with electroweak bosons, Comput. Phys. Commun. 180 (2009) 1661 [arXiv:0811.4559] [INSPIRE].

[30] CMS collaboration, S. Chatrchyan et al., The CMS experiment at the CERN LHC, 2008 JINST 3 S08004 [INSPIRE].

[31] S. Frixione, P. Nason and C. Oleari, Matching NLO QCD computations with Parton Shower simulations: the POWHEG method, JHEP 11 (2007) 070 [arXiv: 0709.2092] [INSPIRE].

[32] F. Maltoni and T. Stelzer, MadEvent: Automatic event generation with MadGraph, JHEP 02 (2003) 027 [hep-ph/0208156] [INSPIRE].

[33] R. Field, Early LHC Underlying Event Data - Findings and Surprises (2010), arXiv: 1010.3558 [INSPIRE].

[34] H.-L. Lai et al., Uncertainty induced by QCD coupling in the CTEQ global analysis of parton distributions, Phys. Rev. D 82 (2010) 054021 [arXiv: 1004.4624] [INSPIRE].

[35] CTEQ collaboration, H. Lai et al., Global QCD analysis of parton structure of the nucleon: CTEQ5 parton distributions, Eur. Phys. J. C 12 (2000) 375 [hep-ph/9903282] [INSPIRE].

[36] G. Bozzi, S. Catani, D. de Florian and M. Grazzini, Transverse-momentum resummation and the spectrum of the Higgs boson at the LHC, Nucl. Phys. B 737 (2006) 73 [hep-ph/0508068] [INSPIRE].

[37] D. de Florian, G. Ferrera, M. Grazzini and D. Tommasini, Transverse-momentum resummation: Higgs boson production at the Tevatron and the LHC, JHEP 11 (2011) 064 [arXiv: 1109.2109] [INSPIRE].

[38] LHC Higgs Cross Section Working Group collaboration, Handbook of LHC Higgs cross sections: 1. Inclusive observables, CERN Report CERN-2011-002, Geneva Switzerland (2011).

[39] LHC Higgs Cross Section Working Group collaboration, Handbook of LHC Higgs cross sections: 2. Differential distributions, CERN Report CERN-2012-002, Geneva Switzerland (2012).

[40] A. Djouadi, J. Kalinowski and M. Spira, HDECAY: A program for Higgs boson decays in the standard model and its supersymmetric extension, Comput. Phys. Commun. 108 (1998) 56 [hep-ph/9704448] [INSPIRE].

[41] A. Djouadi et al., An update of the program HDECAY, in The Les Houches 2009 workshop on TeV colliders: The Tools and Monte Carlo working group Summary Report (2010), [arXiv: 1003.1643] [INSPIRE].

[42] A. Bredenstein, A. Denner, S. Dittmaier and M. Weber, Precise predictions for the Higgs-boson decay $H \rightarrow W W / Z Z \rightarrow 4$ leptons, Phys. Rev. D 74 (2006) 013004 [hep-ph/0604011] [INSPIRE]. 
[43] A. Bredenstein, A. Denner, S. Dittmaier and M. Weber, Radiative corrections to the semileptonic and hadronic Higgs-boson decays $H \rightarrow W W / Z Z \rightarrow 4$ fermions, JHEP 02 (2007) 080 [hep-ph/0611234] [INSPIRE].

[44] S. Actis, G. Passarino, C. Sturm and S. Uccirati, NNLO Computational Techniques: The Cases $H \rightarrow \gamma \gamma$ and $H \rightarrow g g$, Nucl. Phys. B 811 (2009) 182 [arXiv:0809.3667] [InSPIRE].

[45] H.-L. Lai et al., New parton distributions for collider physics, Phys. Rev. D 82 (2010) 074024 [arXiv: 1007.2241] [INSPIRE].

[46] J.M. Campbell and R. Ellis, MCFM for the Tevatron and the LHC, Nucl. Phys. Proc. Suppl. 205-206 (2010) 10 [arXiv: 1007.3492] [INSPIRE].

[47] T. Binoth, N. Kauer and P. Mertsch, Gluon-induced QCD corrections to $\mathrm{pp} \rightarrow \mathrm{ZZ} \rightarrow \ell \bar{\ell} \ell^{\prime} \bar{\ell}^{\prime}$, arXiv: 0807.0024 [INSPIRE].

[48] GEANT4 collaboration, S. Agostinelli et al., GEANT4: A Simulation toolkit, Nucl. Instrum. Meth. A 506 (2003) 250 [INSPIRE].

[49] CMS collaboration, Performance of muon identification in pp collisions at $\sqrt{s}=7 \mathrm{TeV}$, CMS Physics Analysis Summary CMS-PAS-MUO-10-002, Geneva Switzerland (2010).

[50] CMS collaboration, Electron reconstruction and identification at $\sqrt{s}=7 \mathrm{TeV}$, CMS Physics Analysis Summary CMS-PAS-EGM-10-004, Geneva Switzerland (2010).

[51] M. Cacciari and G.P. Salam, Pileup subtraction using jet areas, Phys. Lett. B 659 (2008) 119 [arXiv: 0707.1378] [INSPIRE].

[52] CMS collaboration, Particle-Flow Event Reconstruction in CMS and Performance for Jets, Taus and MET, CMS Physics Analysis Summary CMS-PAS-PFT-09-001, Geneva Switzerland (2009).

[53] CMS collaboration, Jet Performance in pp Collisions at $\sqrt{s}=7 \mathrm{TeV}$, CMS Physics Analysis Summary CMS-PAS-JME-10-003, Geneva Switzerland (2010).

[54] M. Cacciari, G.P. Salam and G. Soyez, The Anti-k(t) jet clustering algorithm, JHEP 04 (2008) 063 [arXiv: 0802.1189] [INSPIRE].

[55] M. Cacciari, G.P. Salam and G. Soyez, FastJet user manual, arXiv:1111.6097 [INSPIRE].

[56] M. Cacciari and G.P. Salam, Dispelling the $N^{3}$ myth for the $k_{t}$ jet-finder, Phys. Lett. B 641 (2006) 57 [hep-ph/0512210] [inSPIRE].

[57] CMS collaboration, Algorithms for b Jet identification in CMS, CMS Physics Analysis Summary CMS-PAS-BTV-09-001, Geneva Switzerland (2009).

[58] CMS collaboration, Performance of the b-jet identification in CMS, CMS Physics Analysis Summary CMS-PAS-BTV-11-001, Geneva Switzerland (2011).

[59] S. Abdullin et al., GARCON: Genetic Algorithm for Rectangular Cut Optimization. User's manual for version 2.0.

[60] K.S. Cranmer, Kernel estimation in high-energy physics, Comput. Phys. Commun. 136 (2001) 198 [hep-ex/0011057] [INSPIRE].

[61] M. Botje et al., The PDF4LHC Working Group Interim Recommendations (2011), arXiv:1101.0538 [INSPIRE].

[62] S. Alekhin et al., The PDF4LHC Working Group Interim Report (2011), arXiv:1101.0536 [INSPIRE]. 
[63] A. Martin, W. Stirling, R. Thorne and G. Watt, Parton distributions for the LHC, Eur. Phys. J. C 63 (2009) 189 [arXiv:0901.0002] [InSPIRE].

[64] NNPDF collaboration, R.D. Ball et al., Impact of Heavy Quark Masses on Parton Distributions and LHC Phenomenology, Nucl. Phys. B 849 (2011) 296 [arXiv:1101.1300] [INSPIRE].

[65] G. Passarino, C. Sturm and S. Uccirati, Higgs Pseudo-Observables, Second Riemann Sheet and All That, Nucl. Phys. B 834 (2010) 77 [arXiv:1001.3360] [InSPIRE].

[66] C. Anastasiou, S. Buehler, F. Herzog and A. Lazopoulos, Total cross-section for Higgs boson hadroproduction with anomalous Standard Model interactions, JHEP 12 (2011) 058 [arXiv: 1107.0683] [INSPIRE].

[67] A.L. Read, Modified frequentist analysis of search results (the CLs method), in 1st Workshop on Confidence Limits, CERN Report CERN-2000-005, Geneva Switzerland (2000).

[68] T. Junk, Confidence level computation for combining searches with small statistics, Nucl. Instrum. Meth. A 434 (1999) 435 [hep-ex/9902006] [INSPIRE].

[69] CMS collaboration, Procedure for the LHC Higgs boson search combination in Summer 2011, CMS Report CMS NOTE 2011-11, Geneva Switzerland (2011).

\section{The CMS collaboration}

\section{Yerevan Physics Institute, Yerevan, Armenia}

S. Chatrchyan, V. Khachatryan, A.M. Sirunyan, A. Tumasyan

\section{Institut für Hochenergiephysik der OeAW, Wien, Austria}

W. Adam, T. Bergauer, M. Dragicevic, J. Erö, C. Fabjan, M. Friedl, R. Frühwirth, V.M. Ghete, J. Hammer ${ }^{1}$, M. Hoch, N. Hörmann, J. Hrubec, M. Jeitler, W. Kiesenhofer, M. Krammer, D. Liko, I. Mikulec, M. Pernicka ${ }^{\dagger}$, B. Rahbaran, C. Rohringer, H. Rohringer, R. Schöfbeck, J. Strauss, A. Taurok, F. Teischinger, P. Wagner, W. Waltenberger, G. Walzel, E. Widl, C.-E. Wulz

National Centre for Particle and High Energy Physics, Minsk, Belarus

V. Mossolov, N. Shumeiko, J. Suarez Gonzalez

\section{Universiteit Antwerpen, Antwerpen, Belgium}

S. Bansal, L. Benucci, T. Cornelis, E.A. De Wolf, X. Janssen, S. Luyckx, T. Maes, L. Mucibello, S. Ochesanu, B. Roland, R. Rougny, M. Selvaggi, H. Van Haevermaet, P. Van Mechelen, N. Van Remortel, A. Van Spilbeeck

\section{Vrije Universiteit Brussel, Brussel, Belgium}

F. Blekman, S. Blyweert, J. D’Hondt, R. Gonzalez Suarez, A. Kalogeropoulos, M. Maes, A. Olbrechts, W. Van Doninck, P. Van Mulders, G.P. Van Onsem, I. Villella

\section{Université Libre de Bruxelles, Bruxelles, Belgium}

O. Charaf, B. Clerbaux, G. De Lentdecker, V. Dero, A.P.R. Gay, G.H. Hammad, T. Hreus, A. Léonard, P.E. Marage, L. Thomas, C. Vander Velde, P. Vanlaer, J. Wickens 
Ghent University, Ghent, Belgium

V. Adler, K. Beernaert, A. Cimmino, S. Costantini, G. Garcia, M. Grunewald, B. Klein, J. Lellouch, A. Marinov, J. Mccartin, A.A. Ocampo Rios, D. Ryckbosch, N. Strobbe, F. Thyssen, M. Tytgat, L. Vanelderen, P. Verwilligen, S. Walsh, E. Yazgan, N. Zaganidis

Université Catholique de Louvain, Louvain-la-Neuve, Belgium

S. Basegmez, G. Bruno, L. Ceard, J. De Favereau De Jeneret, C. Delaere, T. du Pree, D. Favart, L. Forthomme, A. Giammanco ${ }^{2}$, G. Grégoire, J. Hollar, V. Lemaitre, J. Liao, O. Militaru, C. Nuttens, D. Pagano, A. Pin, K. Piotrzkowski, N. Schul

Université de Mons, Mons, Belgium

N. Beliy, T. Caebergs, E. Daubie

Centro Brasileiro de Pesquisas Fisicas, Rio de Janeiro, Brazil

G.A. Alves, M. Correa Martins Junior, D. De Jesus Damiao, T. Martins, M.E. Pol, M.H.G. Souza

Universidade do Estado do Rio de Janeiro, Rio de Janeiro, Brazil

W.L. Aldá Júnior, W. Carvalho, A. Custódio, E.M. Da Costa, C. De Oliveira Martins, S. Fonseca De Souza, D. Matos Figueiredo, L. Mundim, H. Nogima, V. Oguri, W.L. Prado Da Silva, A. Santoro, S.M. Silva Do Amaral, L. Soares Jorge, A. Sznajder

Instituto de Fisica Teorica, Universidade Estadual Paulista, Sao Paulo, Brazil T.S. Anjos ${ }^{3}$, C.A. Bernardes ${ }^{3}$, F.A. Dias ${ }^{4}$, T.R. Fernandez Perez Tomei, E. M. Gregores ${ }^{3}$, C. Lagana, F. Marinho, P.G. Mercadante ${ }^{3}$, S.F. Novaes, Sandra S. Padula

Institute for Nuclear Research and Nuclear Energy, Sofia, Bulgaria

V. Genchev ${ }^{1}$, P. Iaydjiev ${ }^{1}$, S. Piperov, M. Rodozov, S. Stoykova, G. Sultanov, V. Tcholakov, R. Trayanov, M. Vutova

University of Sofia, Sofia, Bulgaria

A. Dimitrov, R. Hadjiiska, A. Karadzhinova, V. Kozhuharov, L. Litov, B. Pavlov, P. Petkov Institute of High Energy Physics, Beijing, China

J.G. Bian, G.M. Chen, H.S. Chen, C.H. Jiang, D. Liang, S. Liang, X. Meng, J. Tao, J. Wang, J. Wang, X. Wang, Z. Wang, H. Xiao, M. Xu, J. Zang, Z. Zhang

State Key Lab. of Nucl. Phys. and Tech., Peking University, Beijing, China C. Asawatangtrakuldee, Y. Ban, S. Guo, Y. Guo, W. Li, S. Liu, Y. Mao, S.J. Qian, H. Teng, S. Wang, B. Zhu, W. Zou

Universidad de Los Andes, Bogota, Colombia

A. Cabrera, B. Gomez Moreno, A.F. Osorio Oliveros, J.C. Sanabria

Technical University of Split, Split, Croatia

N. Godinovic, D. Lelas, R. Plestina ${ }^{5}$, D. Polic, I. Puljak ${ }^{1}$

University of Split, Split, Croatia

Z. Antunovic, M. Dzelalija, M. Kovac

Institute Rudjer Boskovic, Zagreb, Croatia

V. Brigljevic, S. Duric, K. Kadija, J. Luetic, S. Morovic 
University of Cyprus, Nicosia, Cyprus

A. Attikis, M. Galanti, J. Mousa, C. Nicolaou, F. Ptochos, P.A. Razis

Charles University, Prague, Czech Republic

M. Finger, M. Finger Jr.

Academy of Scientific Research and Technology of the Arab Republic of Egypt, Egyptian Network of High Energy Physics, Cairo, Egypt

Y. Assran ${ }^{6}$, A. Ellithi Kamel ${ }^{7}$, S. Khalil ${ }^{8}$, M.A. Mahmoud ${ }^{9}$, A. Radi ${ }^{8,10}$

National Institute of Chemical Physics and Biophysics, Tallinn, Estonia

A. Hektor, M. Kadastik, M. Müntel, M. Raidal, L. Rebane, A. Tiko

Department of Physics, University of Helsinki, Helsinki, Finland

V. Azzolini, P. Eerola, G. Fedi, M. Voutilainen

Helsinki Institute of Physics, Helsinki, Finland

S. Czellar, J. Härkönen, A. Heikkinen, V. Karimäki, R. Kinnunen, M.J. Kortelainen,

T. Lampén, K. Lassila-Perini, S. Lehti, T. Lindén, P. Luukka, T. Mäenpää, T. Peltola,

E. Tuominen, J. Tuominiemi, E. Tuovinen, D. Ungaro, L. Wendland

Lappeenranta University of Technology, Lappeenranta, Finland

K. Banzuzi, A. Korpela, T. Tuuva

Laboratoire d'Annecy-le-Vieux de Physique des Particules, IN2P3-CNRS, Annecy-le-Vieux, France

D. Sillou

DSM/IRFU, CEA/Saclay, Gif-sur-Yvette, France

M. Besancon, S. Choudhury, M. Dejardin, D. Denegri, B. Fabbro, J.L. Faure, F. Ferri,

S. Ganjour, A. Givernaud, P. Gras, G. Hamel de Monchenault, P. Jarry, E. Locci, J. Malcles, L. Millischer, J. Rander, A. Rosowsky, I. Shreyber, M. Titov

Laboratoire Leprince-Ringuet, Ecole Polytechnique, IN2P3-CNRS, Palaiseau, France

S. Baffioni, F. Beaudette, L. Benhabib, L. Bianchini, M. Bluj ${ }^{11}$, C. Broutin, P. Busson,

C. Charlot, N. Daci, T. Dahms, L. Dobrzynski, S. Elgammal, R. Granier de Cassagnac,

M. Haguenauer, P. Miné, C. Mironov, C. Ochando, P. Paganini, D. Sabes, R. Salerno,

Y. Sirois, C. Thiebaux, C. Veelken, A. Zabi

Institut Pluridisciplinaire Hubert Curien, Université de Strasbourg, Université de Haute Alsace Mulhouse, CNRS/IN2P3, Strasbourg, France

J.-L. Agram ${ }^{12}$, J. Andrea, D. Bloch, D. Bodin, J.-M. Brom, M. Cardaci, E.C. Chabert, C. Collard, E. Conte ${ }^{12}$, F. Drouhin ${ }^{12}$, C. Ferro, J.-C. Fontaine ${ }^{12}$, D. Gelé, U. Goerlach, P. Juillot, M. Karim ${ }^{12}$, A.-C. Le Bihan, P. Van Hove

Centre de Calcul de l'Institut National de Physique Nucleaire et de Physique des Particules (IN2P3), Villeurbanne, France

F. Fassi, D. Mercier 
Université de Lyon, Université Claude Bernard Lyon 1, CNRS-IN2P3, Institut de Physique Nucléaire de Lyon, Villeurbanne, France

C. Baty, S. Beauceron, N. Beaupere, M. Bedjidian, O. Bondu, G. Boudoul, D. Boumediene,

H. Brun, J. Chasserat, R. Chierici ${ }^{1}$, D. Contardo, P. Depasse, H. El Mamouni,

A. Falkiewicz, J. Fay, S. Gascon, M. Gouzevitch, B. Ille, T. Kurca, T. Le Grand,

M. Lethuillier, L. Mirabito, S. Perries, V. Sordini, S. Tosi, Y. Tschudi, P. Verdier, S. Viret

Institute of High Energy Physics and Informatization, Tbilisi State University, Tbilisi, Georgia

D. Lomidze

RWTH Aachen University, I. Physikalisches Institut, Aachen, Germany

G. Anagnostou, S. Beranek, M. Edelhoff, L. Feld, N. Heracleous, O. Hindrichs, R. Jussen,

K. Klein, J. Merz, A. Ostapchuk, A. Perieanu, F. Raupach, J. Sammet, S. Schael, D. Sprenger, H. Weber, B. Wittmer, V. Zhukov ${ }^{13}$

RWTH Aachen University, III. Physikalisches Institut A, Aachen, Germany

M. Ata, J. Caudron, E. Dietz-Laursonn, M. Erdmann, A. Güth, T. Hebbeker,

C. Heidemann, K. Hoepfner, T. Klimkovich, D. Klingebiel, P. Kreuzer, D. Lanske ${ }^{\dagger}$, J. Lingemann, C. Magass, M. Merschmeyer, A. Meyer, M. Olschewski, P. Papacz, H. Pieta, H. Reithler, S.A. Schmitz, L. Sonnenschein, J. Steggemann, D. Teyssier, M. Weber

RWTH Aachen University, III. Physikalisches Institut B, Aachen, Germany

M. Bontenackels, V. Cherepanov, M. Davids, G. Flügge, H. Geenen, M. Geisler, W. Haj Ahmad, F. Hoehle, B. Kargoll, T. Kress, Y. Kuessel, A. Linn, A. Nowack, L. Perchalla, O. Pooth, J. Rennefeld, P. Sauerland, A. Stahl, M.H. Zoeller

Deutsches Elektronen-Synchrotron, Hamburg, Germany

M. Aldaya Martin, W. Behrenhoff, U. Behrens, M. Bergholz ${ }^{14}$, A. Bethani, K. Borras,

A. Burgmeier, A. Cakir, L. Calligaris, A. Campbell, E. Castro, D. Dammann, G. Eckerlin,

D. Eckstein, A. Flossdorf, G. Flucke, A. Geiser, J. Hauk, H. Jung ${ }^{1}$, M. Kasemann, P. Katsas, C. Kleinwort, H. Kluge, A. Knutsson, M. Krämer, D. Krücker, E. Kuznetsova, W. Lange, W. Lohmann ${ }^{14}$, B. Lutz, R. Mankel, I. Marfin, M. Marienfeld, I.-A. MelzerPellmann, A.B. Meyer, J. Mnich, A. Mussgiller, S. Naumann-Emme, J. Olzem, A. Petrukhin, D. Pitzl, A. Raspereza, P.M. Ribeiro Cipriano, M. Rosin, J. Salfeld-Nebgen, R. Schmidt ${ }^{14}$, T. Schoerner-Sadenius, N. Sen, A. Spiridonov, M. Stein, J. Tomaszewska, R. Walsh, C. Wissing

University of Hamburg, Hamburg, Germany

C. Autermann, V. Blobel, S. Bobrovskyi, J. Draeger, H. Enderle, J. Erfle, U. Gebbert, M. Görner, T. Hermanns, R.S. Höing, K. Kaschube, G. Kaussen, H. Kirschenmann, R. Klanner, J. Lange, B. Mura, F. Nowak, N. Pietsch, C. Sander, H. Schettler, P. Schleper, E. Schlieckau, A. Schmidt, M. Schröder, T. Schum, H. Stadie, G. Steinbrück, J. Thomsen

Institut für Experimentelle Kernphysik, Karlsruhe, Germany

C. Barth, J. Berger, T. Chwalek, W. De Boer, A. Dierlamm, G. Dirkes, M. Feindt, J. Gruschke, M. Guthoff ${ }^{1}$, C. Hackstein, F. Hartmann, M. Heinrich, H. Held, K.H. Hoffmann, S. Honc, I. Katkov ${ }^{13}$, J.R. Komaragiri, T. Kuhr, D. Martschei, S. Mueller, 
Th. Müller, M. Niegel, A. Nürnberg, O. Oberst, A. Oehler, J. Ott, T. Peiffer, G. Quast, K. Rabbertz, F. Ratnikov, N. Ratnikova, M. Renz, S. Röcker, C. Saout, A. Scheurer, P. Schieferdecker, F.-P. Schilling, M. Schmanau, G. Schott, H.J. Simonis, F.M. Stober,

D. Troendle, J. Wagner-Kuhr, T. Weiler, M. Zeise, E.B. Ziebarth

Institute of Nuclear Physics "Demokritos", Aghia Paraskevi, Greece

G. Daskalakis, T. Geralis, S. Kesisoglou, A. Kyriakis, D. Loukas, I. Manolakos, A. Markou, C. Markou, C. Mavrommatis, E. Ntomari

University of Athens, Athens, Greece

L. Gouskos, T.J. Mertzimekis, A. Panagiotou, N. Saoulidou, E. Stiliaris

University of Ioánnina, Ioánnina, Greece

I. Evangelou, C. Foudas ${ }^{1}$, P. Kokkas, N. Manthos, I. Papadopoulos, V. Patras, F.A. Triantis

KFKI Research Institute for Particle and Nuclear Physics, Budapest, Hungary

A. Aranyi, G. Bencze, L. Boldizsar, C. Hajdu ${ }^{1}$, P. Hidas, D. Horvath ${ }^{15}$, A. Kapusi, K. Krajczar ${ }^{16}$, F. Sikler ${ }^{1}$, V. Veszpremi, G. Vesztergombi ${ }^{16}$

Institute of Nuclear Research ATOMKI, Debrecen, Hungary

N. Beni, J. Molnar, J. Palinkas, Z. Szillasi

University of Debrecen, Debrecen, Hungary

J. Karancsi, P. Raics, Z.L. Trocsanyi, B. Ujvari

Panjab University, Chandigarh, India

S.B. Beri, V. Bhatnagar, N. Dhingra, R. Gupta, M. Jindal, M. Kaur, J.M. Kohli, M.Z. Mehta, N. Nishu, L.K. Saini, A. Sharma, A.P. Singh, J. Singh, S.P. Singh

University of Delhi, Delhi, India

S. Ahuja, B.C. Choudhary, A. Kumar, A. Kumar, S. Malhotra, M. Naimuddin, K. Ranjan, V. Sharma, R.K. Shivpuri

Saha Institute of Nuclear Physics, Kolkata, India

S. Banerjee, S. Bhattacharya, S. Dutta, B. Gomber, S. Jain, S. Jain, R. Khurana, S. Sarkar

Bhabha Atomic Research Centre, Mumbai, India

R.K. Choudhury, D. Dutta, S. Kailas, V. Kumar, A.K. Mohanty ${ }^{1}$, L.M. Pant, P. Shukla

Tata Institute of Fundamental Research - EHEP, Mumbai, India

T. Aziz, S. Ganguly, M. Guchait ${ }^{17}$, A. Gurtu ${ }^{18}$, M. Maity ${ }^{19}$, G. Majumder, K. Mazumdar, G.B. Mohanty, B. Parida, A. Saha, K. Sudhakar, N. Wickramage

Tata Institute of Fundamental Research - HECR, Mumbai, India

S. Banerjee, S. Dugad, N.K. Mondal

Institute for Research in Fundamental Sciences (IPM), Tehran, Iran

H. Arfaei, H. Bakhshiansohi ${ }^{20}$, S.M. Etesami ${ }^{21}$, A. Fahim ${ }^{20}$, M. Hashemi, H. Hesari, A. Jafari ${ }^{20}$, M. Khakzad, A. Mohammadi ${ }^{22}$, M. Mohammadi Najafabadi, S. Paktinat Mehdiabadi, B. Safarzadeh ${ }^{23}$, M. Zeinali ${ }^{21}$

INFN Sezione di Bari ${ }^{a}$, Università di Bari ${ }^{b}$, Politecnico di Bari ${ }^{c}$, Bari, Italy

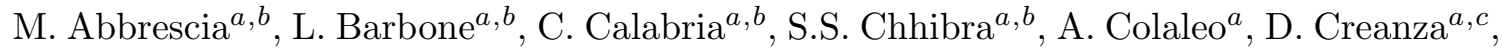


N. De Filippis ${ }^{a, c, 1}$, M. De Palma ${ }^{a, b}$, L. Fiore ${ }^{a}$, G. Iaselli ${ }^{a, c}$, L. Lusito $^{a, b}$, G. Maggi $^{a, c}$, M. Maggi ${ }^{a}$, N. Manna ${ }^{a, b}$, B. Marangelli ${ }^{a, b}$, S. My ${ }^{a, c}$, S. Nuzzo ${ }^{a, b}$, N. Pacifico ${ }^{a, b}$,

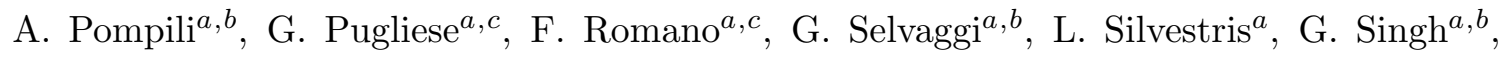
S. Tupputi ${ }^{a, b}$, G. Zito ${ }^{a}$

INFN Sezione di Bologna ${ }^{a}$, Università di Bologna ${ }^{b}$, Bologna, Italy G. Abbiendi ${ }^{a}$, A.C. Benvenuti $a$, D. Bonacorsi ${ }^{a}$, S. Braibant-Giacomelli ${ }^{a}, b$, L. Brigliadori ${ }^{a}$, P. Capiluppi ${ }^{a, b}$, A. Castro ${ }^{a, b}$, F.R. Cavallo ${ }^{a}$, M. Cuffiani ${ }^{a, b}$, G.M. Dallavalle ${ }^{a}$, F. Fabbri ${ }^{a}$, A. Fanfani ${ }^{a}, b$, D. Fasanella ${ }^{a}, 1$, P. Giacomelli ${ }^{a}$, C. Grandi ${ }^{a}$, S. Marcellini ${ }^{a}$,

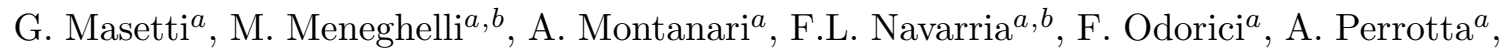
F. Primavera ${ }^{a}$, A.M. Rossi ${ }^{a, b}$, T. Rovelli ${ }^{a, b}$, G. Siroli ${ }^{a, b}$, R. Travaglini ${ }^{a, b}$

INFN Sezione di Catania ${ }^{a}$, Università di Catania ${ }^{b}$, Catania, Italy

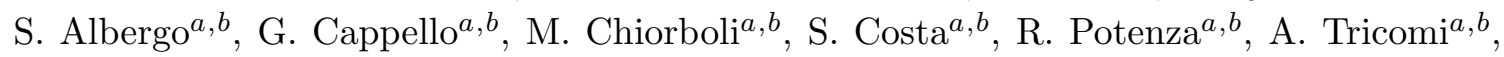
C. Tuve $\mathrm{T}^{a, b}$

INFN Sezione di Firenze ${ }^{a}$, Università di Firenze ${ }^{b}$, Firenze, Italy

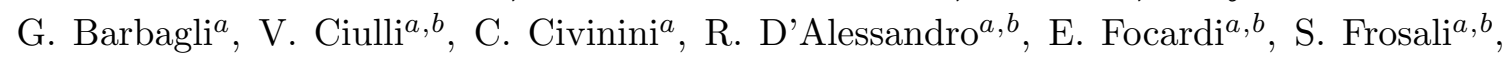
E. Gallo ${ }^{a}$, S. Gonzi ${ }^{a}, b$, M. Meschini ${ }^{a}$, S. Paoletti ${ }^{a}$, G. Sguazzoni ${ }^{a}$, A. Tropiano $^{a, 1}$

INFN Laboratori Nazionali di Frascati, Frascati, Italy

L. Benussi, S. Bianco, S. Colafranceschi ${ }^{24}$, F. Fabbri, D. Piccolo

INFN Sezione di Genova, Genova, Italy

P. Fabbricatore, R. Musenich

INFN Sezione di Milano-Bicocca ${ }^{a}$, Università di Milano-Bicocca ${ }^{b}$, Milano, $^{2}$ Italy

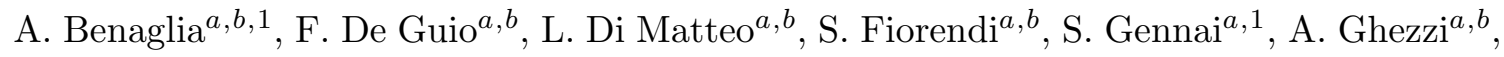
S. Malvezzi ${ }^{a}$, R.A. Manzoni ${ }^{a, b}$, A. Martelli ${ }^{a}, b$, A. Massironi ${ }^{a}, b, 1$, D. Menasce ${ }^{a}$, L. Moroni $^{a}$, M. Paganoni ${ }^{a}, b$ D. Pedrini ${ }^{a}$, S. Ragazzi ${ }^{a, b}$, N. Redaelli ${ }^{a}$, S. Sala ${ }^{a}$, T. Tabarelli de Fatis ${ }^{a}, b$

INFN Sezione di Napoli ${ }^{a}$, Università di Napoli "Federico II" ${ }^{b}$, Napoli, Italy

S. Buontempo ${ }^{a}$, C.A. Carrillo Montoya ${ }^{a, 1}$, N. Cavallo ${ }^{a, 25}$, A. De Cosa ${ }^{a, b}$, O. Dogangun ${ }^{a, b}$, F. Fabozzi ${ }^{a, 25}$, A.O.M. Iorio ${ }^{a, 1}$, L. Lista $^{a}$, M. Merola ${ }^{a, b}$, P. Paolucci $^{a}$

INFN Sezione di Padova ${ }^{a}$, Università di Padova ${ }^{b}$, Università di Trento (Trento) ${ }^{c}$, Padova, Italy

P. Azzi ${ }^{a}$, N. Bacchetta ${ }^{a, 1}$, P. Bellan ${ }^{a, b}$, D. Bisello ${ }^{a, b}$, A. Branca $^{a}$, R. Carlin $^{a, b}$, P. Checchia $^{a}$,

T. Dorigo ${ }^{a}$, U. Dosselli ${ }^{a}$, F. Fanzago ${ }^{a}$, F. Gasparini ${ }^{a, b}$, U. Gasparini ${ }^{a}, b$, A. Gozzelino ${ }^{a}$,

K. Kanishchev, S. Lacaprara ${ }^{a, 26}$, I. Lazzizzera ${ }^{a, c}$, M. Margoni ${ }^{a, b}$, M. Mazzucato ${ }^{a}$, A.T. Meneguzzo ${ }^{a, b}$, M. Nespolo ${ }^{a, 1}$, L. Perrozzi ${ }^{a}$, N. Pozzobon ${ }^{a, b}$, P. Ronchese ${ }^{a, b}$,

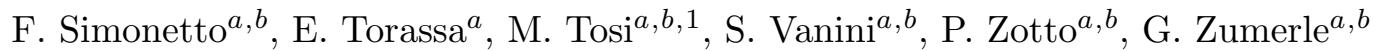

INFN Sezione di Pavia ${ }^{a}$, Università di Pavia ${ }^{b}$, Pavia, Italy

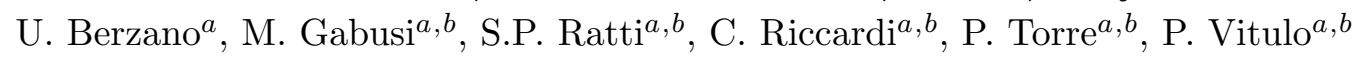

INFN Sezione di Perugia ${ }^{a}$, Università di Perugia ${ }^{b}$, Perugia, Italy

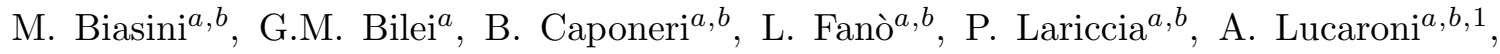


G. Mantovani ${ }^{a, b}$, M. Menichelli $^{a}$, A. Nappi $^{a, b}$, F. Romeo $^{a, b}$, A. Santocchia $^{a, b}$, S. Taroni $^{a, b, 1}$, $^{2}$ M. Valdata ${ }^{a, b}$

INFN Sezione di Pisa ${ }^{a}$, Università di $\mathrm{Pisa}^{b}$, Scuola Normale Superiore di Pisa ${ }^{c}$, Pisa, Italy

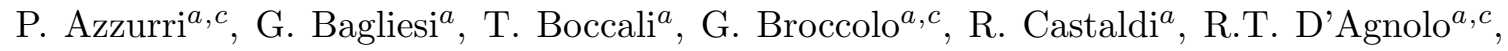

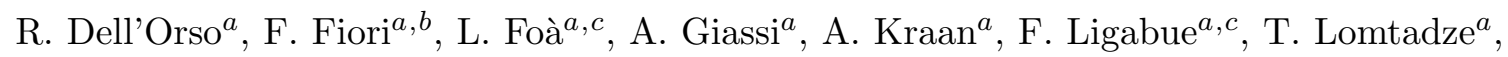
L. Martini ${ }^{a, 27}$, A. Messineo ${ }^{a, b}$, F. Palla ${ }^{a}$, F. Palmonari ${ }^{a}$, A. Rizzi, A.T. Serban ${ }^{a}$,

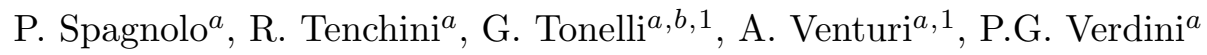

INFN Sezione di Roma ${ }^{a}$, Università di Roma "La Sapienza" ${ }^{b}$, Roma, Italy

L. Barone $e^{a, b}$, F. Cavallari ${ }^{a}$, D. Del Re $e^{a, b, 1}$, M. Diemoz ${ }^{a}$, C. Fanelli, M. Grassi ${ }^{a, 1}$,

E. Longo ${ }^{a, b}$, P. Meridiani ${ }^{a}$, F. Micheli, S. Nourbakhsh ${ }^{a}$, G. Organtini ${ }^{a}, b$, F. Pandolfi ${ }^{a, b}$,

R. Paramatti ${ }^{a}$, S. Rahatlou ${ }^{a, b}$, M. Sigamani ${ }^{a}$, L. Soffi

INFN Sezione di Torino ${ }^{a}$, Università di Torino ${ }^{b}$, Università del Piemonte Orientale (Novara) ${ }^{c}$, Torino, Italy

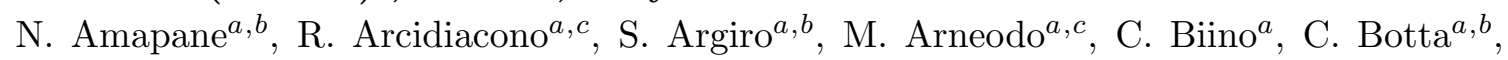
N. Cartiglia ${ }^{a}$, R. Castello ${ }^{a, b}$, M. Costa ${ }^{a, b}$, N. Demaria ${ }^{a}$, A. Graziano ${ }^{a, b}$, C. Mariotti ${ }^{a, 1}$,

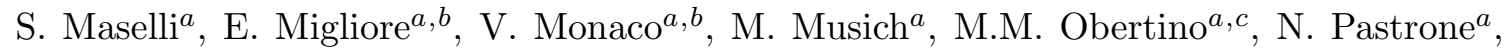

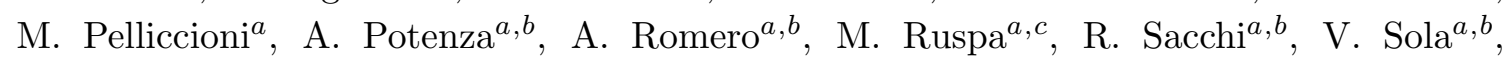
A. Solano ${ }^{a, b}$, A. Staiano ${ }^{a}$, A. Vilela Pereira ${ }^{a}$

INFN Sezione di Trieste ${ }^{a}$, Università di Trieste ${ }^{b}$, Trieste, Italy

S. Belforte ${ }^{a}$, F. Cossutti $^{a}$, G. Della Ricca ${ }^{a, b}$, B. Gobbo ${ }^{a}$, M. Marone $^{a, b}$, D. Montanino $^{a, b, 1}$, A. Penzo ${ }^{a}$

Kangwon National University, Chunchon, Korea

S.G. Heo, S.K. Nam

Kyungpook National University, Daegu, Korea

S. Chang, J. Chung, D.H. Kim, G.N. Kim, J.E. Kim, D.J. Kong, H. Park, S.R. Ro, D.C. Son

Chonnam National University, Institute for Universe and Elementary Particles, Kwangju, Korea

J.Y. Kim, Zero J. Kim, S. Song

Konkuk University, Seoul, Korea

H.Y. Jo

Korea University, Seoul, Korea

S. Choi, D. Gyun, B. Hong, M. Jo, H. Kim, T.J. Kim, K.S. Lee, D.H. Moon, S.K. Park,

E. Seo, K.S. Sim

University of Seoul, Seoul, Korea

M. Choi, S. Kang, H. Kim, J.H. Kim, C. Park, I.C. Park, S. Park, G. Ryu

Sungkyunkwan University, Suwon, Korea

Y. Cho, Y. Choi, Y.K. Choi, J. Goh, M.S. Kim, B. Lee, J. Lee, S. Lee, H. Seo, I. Yu 
Vilnius University, Vilnius, Lithuania

M.J. Bilinskas, I. Grigelionis, M. Janulis

Centro de Investigacion y de Estudios Avanzados del IPN, Mexico City, Mexico

H. Castilla-Valdez, E. De La Cruz-Burelo, I. Heredia-de La Cruz, R. Lopez-Fernandez,

R. Magaña Villalba, J. Martínez-Ortega, A. Sánchez-Hernández, L.M. Villasenor-Cendejas

Universidad Iberoamericana, Mexico City, Mexico

S. Carrillo Moreno, F. Vazquez Valencia

Benemerita Universidad Autonoma de Puebla, Puebla, Mexico

H.A. Salazar Ibarguen

Universidad Autónoma de San Luis Potosí, San Luis Potosí, Mexico

E. Casimiro Linares, A. Morelos Pineda, M.A. Reyes-Santos

University of Auckland, Auckland, New Zealand

D. Krofcheck

University of Canterbury, Christchurch, New Zealand

A.J. Bell, P.H. Butler, R. Doesburg, S. Reucroft, H. Silverwood

National Centre for Physics, Quaid-I-Azam University, Islamabad, Pakistan

M. Ahmad, M.I. Asghar, H.R. Hoorani, S. Khalid, W.A. Khan, T. Khurshid, S. Qazi, M.A. Shah, M. Shoaib

Institute of Experimental Physics, Faculty of Physics, University of Warsaw, Warsaw, Poland

G. Brona, M. Cwiok, W. Dominik, K. Doroba, A. Kalinowski, M. Konecki, J. Krolikowski

Soltan Institute for Nuclear Studies, Warsaw, Poland

H. Bialkowska, B. Boimska, T. Frueboes, R. Gokieli, M. Górski, M. Kazana, K. Nawrocki,

K. Romanowska-Rybinska, M. Szleper, G. Wrochna, P. Zalewski

Laboratório de Instrumentação e Física Experimental de Partículas, Lisboa, Portugal

N. Almeida, P. Bargassa, A. David, P. Faccioli, P.G. Ferreira Parracho, M. Gallinaro,

P. Musella, A. Nayak, J. Pela ${ }^{1}$, P.Q. Ribeiro, J. Seixas, J. Varela, P. Vischia

Joint Institute for Nuclear Research, Dubna, Russia

S. Afanasiev, I. Belotelov, P. Bunin, I. Golutvin, A. Kamenev, V. Karjavin, V. Konoplyanikov, G. Kozlov, A. Lanev, P. Moisenz, V. Palichik, V. Perelygin, M. Savina,

S. Shmatov, V. Smirnov, A. Volodko, A. Zarubin

Petersburg Nuclear Physics Institute, Gatchina (St Petersburg), Russia

S. Evstyukhin, V. Golovtsov, Y. Ivanov, V. Kim, P. Levchenko, V. Murzin, V. Oreshkin,

I. Smirnov, V. Sulimov, L. Uvarov, S. Vavilov, A. Vorobyev, An. Vorobyev

Institute for Nuclear Research, Moscow, Russia

Yu. Andreev, A. Dermenev, S. Gninenko, N. Golubev, M. Kirsanov, N. Krasnikov, V. Matveev, A. Pashenkov, A. Toropin, S. Troitsky 
Institute for Theoretical and Experimental Physics, Moscow, Russia

V. Epshteyn, M. Erofeeva, V. Gavrilov, M. Kossov ${ }^{1}$, A. Krokhotin, N. Lychkovskaya, V. Popov, G. Safronov, S. Semenov, V. Stolin, E. Vlasov, A. Zhokin

Moscow State University, Moscow, Russia

A. Belyaev, E. Boos, M. Dubinin ${ }^{4}$, L. Dudko, A. Ershov, A. Gribushin, O. Kodolova, I. Lokhtin, A. Markina, S. Obraztsov, M. Perfilov, S. Petrushanko, L. Sarycheva ${ }^{\dagger}$, V. Savrin, A. Snigirev

P.N. Lebedev Physical Institute, Moscow, Russia

V. Andreev, M. Azarkin, I. Dremin, M. Kirakosyan, A. Leonidov, G. Mesyats, S.V. Rusakov, A. Vinogradov

State Research Center of Russian Federation, Institute for High Energy Physics, Protvino, Russia

I. Azhgirey, I. Bayshev, S. Bitioukov, V. Grishin ${ }^{1}$, V. Kachanov, D. Konstantinov,

A. Korablev, V. Krychkine, V. Petrov, R. Ryutin, A. Sobol, L. Tourtchanovitch, S. Troshin,

N. Tyurin, A. Uzunian, A. Volkov

University of Belgrade, Faculty of Physics and Vinca Institute of Nuclear Sciences, Belgrade, Serbia

P. Adzic ${ }^{28}$, M. Djordjevic, M. Ekmedzic, D. Krpic ${ }^{28}$, J. Milosevic

Centro de Investigaciones Energéticas Medioambientales y Tecnológicas (CIEMAT), Madrid, Spain

M. Aguilar-Benitez, J. Alcaraz Maestre, P. Arce, C. Battilana, E. Calvo, M. Cerrada, M. Chamizo Llatas, N. Colino, B. De La Cruz, A. Delgado Peris, C. Diez Pardos,

D. Domínguez Vázquez, C. Fernandez Bedoya, J.P. Fernández Ramos, A. Ferrando, J. Flix, M.C. Fouz, P. Garcia-Abia, O. Gonzalez Lopez, S. Goy Lopez, J.M. Hernandez, M.I. Josa, G. Merino, J. Puerta Pelayo, I. Redondo, L. Romero, J. Santaolalla, M.S. Soares, C. Willmott

Universidad Autónoma de Madrid, Madrid, Spain

C. Albajar, G. Codispoti, J.F. de Trocóniz

Universidad de Oviedo, Oviedo, Spain

J. Cuevas, J. Fernandez Menendez, S. Folgueras, I. Gonzalez Caballero, L. Lloret Iglesias, J. Piedra Gomez ${ }^{29}$, J.M. Vizan Garcia

Instituto de Física de Cantabria (IFCA), CSIC-Universidad de Cantabria, Santander, Spain

J.A. Brochero Cifuentes, I.J. Cabrillo, A. Calderon, S.H. Chuang, J. Duarte Campderros, M. Felcini ${ }^{30}$, M. Fernandez, G. Gomez, J. Gonzalez Sanchez, C. Jorda, P. Lobelle Pardo, A. Lopez Virto, J. Marco, R. Marco, C. Martinez Rivero, F. Matorras, F.J. Munoz Sanchez,

T. Rodrigo, A.Y. Rodríguez-Marrero, A. Ruiz-Jimeno, L. Scodellaro, M. Sobron Sanudo, I. Vila, R. Vilar Cortabitarte

CERN, European Organization for Nuclear Research, Geneva, Switzerland D. Abbaneo, E. Auffray, G. Auzinger, P. Baillon, A.H. Ball, D. Barney, C. Bernet ${ }^{5}$, 
W. Bialas, G. Bianchi, P. Bloch, A. Bocci, H. Breuker, K. Bunkowski, T. Camporesi, G. Cerminara, T. Christiansen, J.A. Coarasa Perez, B. Curé, D. D'Enterria, A. De Roeck, S. Di Guida, M. Dobson, N. Dupont-Sagorin, A. Elliott-Peisert, B. Frisch, W. Funk, A. Gaddi, G. Georgiou, H. Gerwig, M. Giffels, D. Gigi, K. Gill, D. Giordano, M. Giunta, F. Glege, R. Gomez-Reino Garrido, P. Govoni, S. Gowdy, R. Guida, L. Guiducci, M. Hansen, P. Harris, C. Hartl, J. Harvey, B. Hegner, A. Hinzmann, H.F. Hoffmann, V. Innocente, P. Janot, K. Kaadze, E. Karavakis, K. Kousouris, P. Lecoq, P. Lenzi, C. Lourenço, T. Mäki, M. Malberti, L. Malgeri, M. Mannelli, L. Masetti, G. Mavromanolakis, F. Meijers, S. Mersi, E. Meschi, R. Moser, M.U. Mozer, M. Mulders, E. Nesvold, M. Nguyen, T. Orimoto, L. Orsini, E. Palencia Cortezon, E. Perez, A. Petrilli, A. Pfeiffer, M. Pierini, M. Pimiä, D. Piparo, G. Polese, L. Quertenmont, A. Racz, W. Reece, J. Rodrigues Antunes, G. Rolandi ${ }^{31}$, T. Rommerskirchen, C. Rovelli ${ }^{32}$, M. Rovere, H. Sakulin, F. Santanastasio, C. Schäfer, C. Schwick, I. Segoni, A. Sharma, P. Siegrist, P. Silva, M. Simon, P. Sphicas ${ }^{33}$, D. Spiga, M. Spiropulu ${ }^{4}$, M. Stoye, A. Tsirou, G.I. Veres ${ }^{16}$, P. Vichoudis, H.K. Wöhri, S.D. Worm ${ }^{34}$, W.D. Zeuner

\section{Paul Scherrer Institut, Villigen, Switzerland}

W. Bertl, K. Deiters, W. Erdmann, K. Gabathuler, R. Horisberger, Q. Ingram, H.C. Kaestli, S. König, D. Kotlinski, U. Langenegger, F. Meier, D. Renker, T. Rohe, J. Sibille ${ }^{35}$

\section{Institute for Particle Physics, ETH Zurich, Zurich, Switzerland}

L. Bäni, P. Bortignon, M.A. Buchmann, B. Casal, N. Chanon, Z. Chen, A. Deisher, G. Dissertori, M. Dittmar, M. Dünser, J. Eugster, K. Freudenreich, C. Grab, P. Lecomte, W. Lustermann, P. Martinez Ruiz del Arbol, N. Mohr, F. Moortgat, C. Nägeli ${ }^{36}$, P. Nef, F. Nessi-Tedaldi, L. Pape, F. Pauss, M. Peruzzi, F.J. Ronga, M. Rossini, L. Sala, A.K. Sanchez, M.-C. Sawley, A. Starodumov ${ }^{37}$, B. Stieger, M. Takahashi, L. Tauscher ${ }^{\dagger}$, A. Thea, K. Theofilatos, D. Treille, C. Urscheler, R. Wallny, H.A. Weber, L. Wehrli, J. Weng

\section{Universität Zürich, Zurich, Switzerland}

E. Aguilo, C. Amsler, V. Chiochia, S. De Visscher, C. Favaro, M. Ivova Rikova, B. Millan Mejias, P. Otiougova, P. Robmann, H. Snoek, M. Verzetti

\section{National Central University, Chung-Li, Taiwan}

Y.H. Chang, K.H. Chen, C.M. Kuo, S.W. Li, W. Lin, Z.K. Liu, Y.J. Lu, D. Mekterovic, R. Volpe, S.S. Yu

\section{National Taiwan University (NTU), Taipei, Taiwan}

P. Bartalini, P. Chang, Y.H. Chang, Y.W. Chang, Y. Chao, K.F. Chen, C. Dietz, U. Grundler, W.-S. Hou, Y. Hsiung, K.Y. Kao, Y.J. Lei, R.-S. Lu, D. Majumder, E. Petrakou, X. Shi, J.G. Shiu, Y.M. Tzeng, M. Wang

\section{Cukurova University, Adana, Turkey}

A. Adiguzel, M.N. Bakirci ${ }^{38}$, S. Cerci ${ }^{39}$, C. Dozen, I. Dumanoglu, E. Eskut, S. Girgis, G. Gokbulut, I. Hos, E.E. Kangal, G. Karapinar, A. Kayis Topaksu, G. Onengut, K. Ozdemir, S. Ozturk ${ }^{40}$, A. Polatoz, K. Sogut ${ }^{41}$, D. Sunar Cerci ${ }^{39}$, B. Tali ${ }^{39}$, H. Topakli ${ }^{38}$, D. Uzun, L.N. Vergili, M. Vergili 
Middle East Technical University, Physics Department, Ankara, Turkey I.V. Akin, T. Aliev, B. Bilin, S. Bilmis, M. Deniz, H. Gamsizkan, A.M. Guler, K. Ocalan, A. Ozpineci, M. Serin, R. Sever, U.E. Surat, M. Yalvac, E. Yildirim, M. Zeyrek

Bogazici University, Istanbul, Turkey

M. Deliomeroglu, E. Gülmez, B. Isildak, M. Kaya ${ }^{42}$, O. Kaya ${ }^{42}$, S. Ozkorucuklu ${ }^{43}$, N. Sonmez ${ }^{44}$

National Scientific Center, Kharkov Institute of Physics and Technology, Kharkov, Ukraine

L. Levchuk

\section{University of Bristol, Bristol, United Kingdom}

F. Bostock, J.J. Brooke, E. Clement, D. Cussans, H. Flacher, R. Frazier, J. Goldstein, M. Grimes, G.P. Heath, H.F. Heath, L. Kreczko, S. Metson, D.M. Newbold ${ }^{34}$, K. Nirunpong, A. Poll, S. Senkin, V.J. Smith, T. Williams

\section{Rutherford Appleton Laboratory, Didcot, United Kingdom}

L. Basso ${ }^{45}$, K.W. Bell, A. Belyaev ${ }^{45}$, C. Brew, R.M. Brown, D.J.A. Cockerill, J.A. Coughlan, K. Harder, S. Harper, J. Jackson, B.W. Kennedy, E. Olaiya, D. Petyt, B.C. Radburn-Smith, C.H. Shepherd-Themistocleous, I.R. Tomalin, W.J. Womersley

\section{Imperial College, London, United Kingdom}

R. Bainbridge, G. Ball, R. Beuselinck, O. Buchmuller, D. Colling, N. Cripps, M. Cutajar, P. Dauncey, G. Davies, M. Della Negra, W. Ferguson, J. Fulcher, D. Futyan, A. Gilbert, A. Guneratne Bryer, G. Hall, Z. Hatherell, J. Hays, G. Iles, M. Jarvis, G. Karapostoli, L. Lyons, A.-M. Magnan, J. Marrouche, B. Mathias, R. Nandi, J. Nash, A. Nikitenko ${ }^{37}$, A. Papageorgiou, M. Pesaresi, K. Petridis, M. Pioppi ${ }^{46}$, D.M. Raymond, S. Rogerson, N. Rompotis, A. Rose, M.J. Ryan, C. Seez, A. Sparrow, A. Tapper, S. Tourneur, M. Vazquez Acosta, T. Virdee, S. Wakefield, N. Wardle, D. Wardrope, T. Whyntie

\section{Brunel University, Uxbridge, United Kingdom}

M. Barrett, M. Chadwick, J.E. Cole, P.R. Hobson, A. Khan, P. Kyberd, D. Leslie, W. Martin, I.D. Reid, P. Symonds, L. Teodorescu, M. Turner

Baylor University, Waco, USA

K. Hatakeyama, H. Liu, T. Scarborough

The University of Alabama, Tuscaloosa, USA

C. Henderson

\section{Boston University, Boston, USA}

A. Avetisyan, T. Bose, E. Carrera Jarrin, C. Fantasia, A. Heister, J. St. John, P. Lawson, D. Lazic, J. Rohlf, D. Sperka, L. Sulak

Brown University, Providence, USA

S. Bhattacharya, D. Cutts, A. Ferapontov, U. Heintz, S. Jabeen, G. Kukartsev, G. Landsberg, M. Luk, M. Narain, D. Nguyen, M. Segala, T. Sinthuprasith, T. Speer, K.V. Tsang 
University of California, Davis, Davis, USA

R. Breedon, G. Breto, M. Calderon De La Barca Sanchez, M. Caulfield, S. Chauhan, M. Chertok, J. Conway, R. Conway, P.T. Cox, J. Dolen, R. Erbacher, M. Gardner, R. Houtz, W. Ko, A. Kopecky, R. Lander, O. Mall, T. Miceli, R. Nelson, D. Pellett, J. Robles, B. Rutherford, M. Searle, J. Smith, M. Squires, M. Tripathi, R. Vasquez Sierra

University of California, Los Angeles, Los Angeles, USA

V. Andreev, K. Arisaka, D. Cline, R. Cousins, J. Duris, S. Erhan, P. Everaerts, C. Farrell, J. Hauser, M. Ignatenko, C. Jarvis, C. Plager, G. Rakness, P. Schlein ${ }^{\dagger}$, J. Tucker, V. Valuev, M. Weber

\section{University of California, Riverside, Riverside, USA}

J. Babb, R. Clare, J. Ellison, J.W. Gary, F. Giordano, G. Hanson, G.Y. Jeng, H. Liu, O.R. Long, A. Luthra, H. Nguyen, S. Paramesvaran, J. Sturdy, S. Sumowidagdo, R. Wilken, S. Wimpenny

\section{University of California, San Diego, La Jolla, USA}

W. Andrews, J.G. Branson, G.B. Cerati, S. Cittolin, D. Evans, F. Golf, A. Holzner, R. Kelley, M. Lebourgeois, J. Letts, I. Macneill, B. Mangano, S. Padhi, C. Palmer, G. Petrucciani, H. Pi, M. Pieri, R. Ranieri, M. Sani, I. Sfiligoi, V. Sharma, S. Simon, E. Sudano, M. Tadel, Y. Tu, A. Vartak, S. Wasserbaech ${ }^{47}$, F. Würthwein, A. Yagil, J. Yoo

University of California, Santa Barbara, Santa Barbara, USA

D. Barge, R. Bellan, C. Campagnari, M. D’Alfonso, T. Danielson, K. Flowers, P. Geffert, J. Incandela, C. Justus, P. Kalavase, S.A. Koay, D. Kovalskyi ${ }^{1}$, V. Krutelyov, S. Lowette, N. Mccoll, V. Pavlunin, F. Rebassoo, J. Ribnik, J. Richman, R. Rossin, D. Stuart, W. To, J.R. Vlimant, C. West

\section{California Institute of Technology, Pasadena, USA}

A. Apresyan, A. Bornheim, J. Bunn, Y. Chen, E. Di Marco, J. Duarte, M. Gataullin, Y. Ma, A. Mott, H.B. Newman, C. Rogan, V. Timciuc, P. Traczyk, J. Veverka, R. Wilkinson, Y. Yang, R.Y. Zhu

\section{Carnegie Mellon University, Pittsburgh, USA}

B. Akgun, R. Carroll, T. Ferguson, Y. Iiyama, D.W. Jang, S.Y. Jun, Y.F. Liu, M. Paulini, J. Russ, H. Vogel, I. Vorobiev

\section{University of Colorado at Boulder, Boulder, USA}

J.P. Cumalat, M.E. Dinardo, B.R. Drell, C.J. Edelmaier, W.T. Ford, A. Gaz, B. Heyburn, E. Luiggi Lopez, U. Nauenberg, J.G. Smith, K. Stenson, K.A. Ulmer, S.R. Wagner, S.L. Zang

\section{Cornell University, Ithaca, USA}

L. Agostino, J. Alexander, A. Chatterjee, N. Eggert, L.K. Gibbons, B. Heltsley, W. Hopkins, A. Khukhunaishvili, B. Kreis, N. Mirman, G. Nicolas Kaufman, J.R. Patterson, A. Ryd, E. Salvati, W. Sun, W.D. Teo, J. Thom, J. Thompson, J. Vaughan, Y. Weng, L. Winstrom, P. Wittich 


\section{Fairfield University, Fairfield, USA}

A. Biselli, D. Winn

\section{Fermi National Accelerator Laboratory, Batavia, USA}

S. Abdullin, M. Albrow, J. Anderson, G. Apollinari, M. Atac, J.A. Bakken, L.A.T. Bauerdick, A. Beretvas, J. Berryhill, P.C. Bhat, I. Bloch, K. Burkett, J.N. Butler, V. Chetluru, H.W.K. Cheung, F. Chlebana, S. Cihangir, W. Cooper, D.P. Eartly, V.D. Elvira, S. Esen, I. Fisk, J. Freeman, Y. Gao, E. Gottschalk, D. Green, O. Gutsche, J. Hanlon, R.M. Harris, J. Hirschauer, B. Hooberman, H. Jensen, S. Jindariani, M. Johnson, U. Joshi, B. Klima, S. Kunori, S. Kwan, C. Leonidopoulos, D. Lincoln, R. Lipton, J. Lykken, K. Maeshima, J.M. Marraffino, S. Maruyama, D. Mason, P. McBride, T. Miao, K. Mishra, S. Mrenna, Y. Musienko ${ }^{48}$, C. Newman-Holmes, V. O’Dell, J. Pivarski, R. Pordes, O. Prokofyev, T. Schwarz, E. Sexton-Kennedy, S. Sharma, W.J. Spalding, L. Spiegel, P. Tan, L. Taylor, S. Tkaczyk, L. Uplegger, E.W. Vaandering, R. Vidal, J. Whitmore, W. Wu, F. Yang, F. Yumiceva, J.C. Yun

University of Florida, Gainesville, USA

D. Acosta, P. Avery, D. Bourilkov, M. Chen, S. Das, M. De Gruttola, G.P. Di Giovanni, D. Dobur, A. Drozdetskiy, R.D. Field, M. Fisher, Y. Fu, I.K. Furic, J. Gartner, S. Goldberg, J. Hugon, B. Kim, J. Konigsberg, A. Korytov, A. Kropivnitskaya, T. Kypreos, J.F. Low, K. Matchev, P. Milenovic ${ }^{49}$, G. Mitselmakher, L. Muniz, R. Remington, A. Rinkevicius, M. Schmitt, B. Scurlock, P. Sellers, N. Skhirtladze, M. Snowball, D. Wang, J. Yelton, M. Zakaria

\section{Florida International University, Miami, USA}

V. Gaultney, L.M. Lebolo, S. Linn, P. Markowitz, G. Martinez, J.L. Rodriguez

\section{Florida State University, Tallahassee, USA}

T. Adams, A. Askew, J. Bochenek, J. Chen, B. Diamond, S.V. Gleyzer, J. Haas,

S. Hagopian, V. Hagopian, M. Jenkins, K.F. Johnson, H. Prosper, S. Sekmen, V. Veeraraghavan, M. Weinberg

Florida Institute of Technology, Melbourne, USA

M.M. Baarmand, B. Dorney, M. Hohlmann, H. Kalakhety, I. Vodopiyanov

\section{University of Illinois at Chicago (UIC), Chicago, USA}

M.R. Adams, I.M. Anghel, L. Apanasevich, Y. Bai, V.E. Bazterra, R.R. Betts, J. Callner, R. Cavanaugh, C. Dragoiu, L. Gauthier, C.E. Gerber, D.J. Hofman, S. Khalatyan, G.J. Kunde ${ }^{50}$, F. Lacroix, M. Malek, C. O'Brien, C. Silkworth, C. Silvestre, D. Strom, N. Varelas

\section{The University of Iowa, Iowa City, USA}

U. Akgun, E.A. Albayrak, B. Bilki ${ }^{51}$, W. Clarida, F. Duru, S. Griffiths, C.K. Lae, E. McCliment, J.-P. Merlo, H. Mermerkaya ${ }^{52}$, A. Mestvirishvili, A. Moeller, J. Nachtman, C.R. Newsom, E. Norbeck, J. Olson, Y. Onel, F. Ozok, S. Sen, E. Tiras, J. Wetzel, T. Yetkin, K. Yi 
Johns Hopkins University, Baltimore, USA

B.A. Barnett, B. Blumenfeld, S. Bolognesi, A. Bonato, D. Fehling, G. Giurgiu, A.V. Gritsan, Z.J. Guo, G. Hu, P. Maksimovic, S. Rappoccio, M. Swartz, N.V. Tran, A. Whitbeck

\section{The University of Kansas, Lawrence, USA}

P. Baringer, A. Bean, G. Benelli, O. Grachov, R.P. Kenny Iii, M. Murray, D. Noonan, S. Sanders, R. Stringer, G. Tinti, J.S. Wood, V. Zhukova

Kansas State University, Manhattan, USA

A.F. Barfuss, T. Bolton, I. Chakaberia, A. Ivanov, S. Khalil, M. Makouski, Y. Maravin, S. Shrestha, I. Svintradze

Lawrence Livermore National Laboratory, Livermore, USA

J. Gronberg, D. Lange, D. Wright

University of Maryland, College Park, USA

A. Baden, M. Boutemeur, B. Calvert, S.C. Eno, J.A. Gomez, N.J. Hadley, R.G. Kellogg, M. Kirn, T. Kolberg, Y. Lu, M. Marionneau, A.C. Mignerey, A. Peterman, K. Rossato, P. Rumerio, A. Skuja, J. Temple, M.B. Tonjes, S.C. Tonwar, E. Twedt

\section{Massachusetts Institute of Technology, Cambridge, USA}

B. Alver, G. Bauer, J. Bendavid, W. Busza, E. Butz, I.A. Cali, M. Chan, V. Dutta, G. Gomez Ceballos, M. Goncharov, K.A. Hahn, Y. Kim, M. Klute, Y.-J. Lee, W. Li, P.D. Luckey, T. Ma, S. Nahn, C. Paus, D. Ralph, C. Roland, G. Roland, M. Rudolph, G.S.F. Stephans, F. Stöckli, K. Sumorok, K. Sung, D. Velicanu, E.A. Wenger, R. Wolf, B. Wyslouch, S. Xie, M. Yang, Y. Yilmaz, A.S. Yoon, M. Zanetti

University of Minnesota, Minneapolis, USA

S.I. Cooper, P. Cushman, B. Dahmes, A. De Benedetti, G. Franzoni, A. Gude, J. Haupt, S.C. Kao, K. Klapoetke, Y. Kubota, J. Mans, N. Pastika, V. Rekovic, R. Rusack, M. Sasseville, A. Singovsky, N. Tambe, J. Turkewitz

University of Mississippi, University, USA

L.M. Cremaldi, R. Godang, R. Kroeger, L. Perera, R. Rahmat, D.A. Sanders, D. Summers

University of Nebraska-Lincoln, Lincoln, USA

E. Avdeeva, K. Bloom, S. Bose, J. Butt, D.R. Claes, A. Dominguez, M. Eads, P. Jindal, J. Keller, I. Kravchenko, J. Lazo-Flores, H. Malbouisson, S. Malik, G.R. Snow

State University of New York at Buffalo, Buffalo, USA

U. Baur, A. Godshalk, I. Iashvili, S. Jain, A. Kharchilava, A. Kumar, S.P. Shipkowski, K. Smith, Z. Wan

Northeastern University, Boston, USA

G. Alverson, E. Barberis, D. Baumgartel, M. Chasco, D. Trocino, D. Wood, J. Zhang

Northwestern University, Evanston, USA

A. Anastassov, A. Kubik, N. Mucia, N. Odell, R.A. Ofierzynski, B. Pollack, A. Pozdnyakov, M. Schmitt, S. Stoynev, M. Velasco, S. Won 
University of Notre Dame, Notre Dame, USA

L. Antonelli, D. Berry, A. Brinkerhoff, M. Hildreth, C. Jessop, D.J. Karmgard, J. Kolb,

K. Lannon, W. Luo, S. Lynch, N. Marinelli, D.M. Morse, T. Pearson, R. Ruchti, J. Slaunwhite, N. Valls, M. Wayne, M. Wolf, J. Ziegler

The Ohio State University, Columbus, USA

B. Bylsma, L.S. Durkin, C. Hill, P. Killewald, K. Kotov, T.Y. Ling, D. Puigh, M. Rodenburg, C. Vuosalo, G. Williams

\section{Princeton University, Princeton, USA}

N. Adam, E. Berry, P. Elmer, D. Gerbaudo, V. Halyo, P. Hebda, J. Hegeman, A. Hunt, E. Laird, D. Lopes Pegna, P. Lujan, D. Marlow, T. Medvedeva, M. Mooney, J. Olsen, P. Piroué, X. Quan, A. Raval, H. Saka, D. Stickland, C. Tully, J.S. Werner, A. Zuranski

\section{University of Puerto Rico, Mayaguez, USA}

J.G. Acosta, X.T. Huang, A. Lopez, H. Mendez, S. Oliveros, J.E. Ramirez Vargas, A. Zatserklyaniy

Purdue University, West Lafayette, USA

E. Alagoz, V.E. Barnes, D. Benedetti, G. Bolla, D. Bortoletto, M. De Mattia, A. Everett, L. Gutay, Z. Hu, M. Jones, O. Koybasi, M. Kress, A.T. Laasanen, N. Leonardo, V. Maroussov, P. Merkel, D.H. Miller, N. Neumeister, I. Shipsey, D. Silvers, A. Svyatkovskiy, M. Vidal Marono, H.D. Yoo, J. Zablocki, Y. Zheng

Purdue University Calumet, Hammond, USA

S. Guragain, N. Parashar

Rice University, Houston, USA

A. Adair, C. Boulahouache, V. Cuplov, K.M. Ecklund, F.J.M. Geurts, B.P. Padley, R. Redjimi, J. Roberts, J. Zabel

University of Rochester, Rochester, USA

B. Betchart, A. Bodek, Y.S. Chung, R. Covarelli, P. de Barbaro, R. Demina, Y. Eshaq, A. Garcia-Bellido, P. Goldenzweig, Y. Gotra, J. Han, A. Harel, D.C. Miner, G. Petrillo, W. Sakumoto, D. Vishnevskiy, M. Zielinski

The Rockefeller University, New York, USA

A. Bhatti, R. Ciesielski, L. Demortier, K. Goulianos, G. Lungu, S. Malik, C. Mesropian

Rutgers, the State University of New Jersey, Piscataway, USA

S. Arora, O. Atramentov, A. Barker, J.P. Chou, C. Contreras-Campana, E. ContrerasCampana, D. Duggan, D. Ferencek, Y. Gershtein, R. Gray, E. Halkiadakis, D. Hidas, D. Hits, A. Lath, S. Panwalkar, M. Park, R. Patel, A. Richards, K. Rose, S. Salur, S. Schnetzer, C. Seitz, S. Somalwar, R. Stone, S. Thomas

University of Tennessee, Knoxville, USA

G. Cerizza, M. Hollingsworth, S. Spanier, Z.C. Yang, A. York

Texas A\&M University, College Station, USA

R. Eusebi, W. Flanagan, J. Gilmore, T. Kamon ${ }^{53}$, V. Khotilovich, R. Montalvo, 
I. Osipenkov, Y. Pakhotin, A. Perloff, J. Roe, A. Safonov, T. Sakuma, S. Sengupta, I. Suarez, A. Tatarinov, D. Toback

\section{Texas Tech University, Lubbock, USA}

N. Akchurin, C. Bardak, J. Damgov, P.R. Dudero, C. Jeong, K. Kovitanggoon, S.W. Lee, T. Libeiro, P. Mane, Y. Roh, A. Sill, I. Volobouev, R. Wigmans

\section{Vanderbilt University, Nashville, USA}

E. Appelt, E. Brownson, D. Engh, C. Florez, W. Gabella, A. Gurrola, M. Issah, W. Johns, P. Kurt, C. Maguire, A. Melo, P. Sheldon, B. Snook, S. Tuo, J. Velkovska

\section{University of Virginia, Charlottesville, USA}

M.W. Arenton, M. Balazs, S. Boutle, S. Conetti, B. Cox, B. Francis, S. Goadhouse, J. Goodell, R. Hirosky, A. Ledovskoy, C. Lin, C. Neu, J. Wood, R. Yohay

\section{Wayne State University, Detroit, USA}

S. Gollapinni, R. Harr, P.E. Karchin, C. Kottachchi Kankanamge Don, P. Lamichhane, M. Mattson, C. Milstène, A. Sakharov

\section{University of Wisconsin, Madison, USA}

M. Anderson, M. Bachtis, D. Belknap, J.N. Bellinger, J. Bernardini, L. Borrello, D. Carlsmith, M. Cepeda, S. Dasu, J. Efron, E. Friis, L. Gray, K.S. Grogg, M. Grothe, R. Hall-Wilton, M. Herndon, A. Hervé, P. Klabbers, J. Klukas, A. Lanaro, C. Lazaridis, J. Leonard, R. Loveless, A. Mohapatra, I. Ojalvo, G.A. Pierro, I. Ross, A. Savin, W.H. Smith, J. Swanson

$\dagger$ : Deceased

1: Also at CERN, European Organization for Nuclear Research, Geneva, Switzerland

2: Also at National Institute of Chemical Physics and Biophysics, Tallinn, Estonia

3: Also at Universidade Federal do ABC, Santo Andre, Brazil

4: Also at California Institute of Technology, Pasadena, USA

5: Also at Laboratoire Leprince-Ringuet, Ecole Polytechnique, IN2P3-CNRS, Palaiseau, France

6: Also at Suez Canal University, Suez, Egypt

7: Also at Cairo University, Cairo, Egypt

8: Also at British University, Cairo, Egypt

9: Also at Fayoum University, El-Fayoum, Egypt

10: Now at Ain Shams University, Cairo, Egypt

11: Also at Soltan Institute for Nuclear Studies, Warsaw, Poland

12: Also at Université de Haute-Alsace, Mulhouse, France

13: Also at Moscow State University, Moscow, Russia

14: Also at Brandenburg University of Technology, Cottbus, Germany

15: Also at Institute of Nuclear Research ATOMKI, Debrecen, Hungary

16: Also at Eötvös Loránd University, Budapest, Hungary

17: Also at Tata Institute of Fundamental Research - HECR, Mumbai, India

18: Now at King Abdulaziz University, Jeddah, Saudi Arabia

19: Also at University of Visva-Bharati, Santiniketan, India

20: Also at Sharif University of Technology, Tehran, Iran

21: Also at Isfahan University of Technology, Isfahan, Iran 
22: Also at Shiraz University, Shiraz, Iran

23: Also at Plasma Physics Research Center, Science and Research Branch, Islamic Azad University, Teheran, Iran

24: Also at Facoltà Ingegneria Università di Roma, Roma, Italy

25: Also at Università della Basilicata, Potenza, Italy

26: Also at Laboratori Nazionali di Legnaro dell' INFN, Legnaro, Italy

27: Also at Università degli studi di Siena, Siena, Italy

28: Also at Faculty of Physics of University of Belgrade, Belgrade, Serbia

29: Also at University of Florida, Gainesville, USA

30: Also at University of California, Los Angeles, Los Angeles, USA

31: Also at Scuola Normale e Sezione dell' INFN, Pisa, Italy

32: Also at INFN Sezione di Roma; Università di Roma "La Sapienza", Roma, Italy

33: Also at University of Athens, Athens, Greece

34: Also at Rutherford Appleton Laboratory, Didcot, United Kingdom

35: Also at The University of Kansas, Lawrence, USA

36: Also at Paul Scherrer Institut, Villigen, Switzerland

37: Also at Institute for Theoretical and Experimental Physics, Moscow, Russia

38: Also at Gaziosmanpasa University, Tokat, Turkey

39: Also at Adiyaman University, Adiyaman, Turkey

40: Also at The University of Iowa, Iowa City, USA

41: Also at Mersin University, Mersin, Turkey

42: Also at Kafkas University, Kars, Turkey

43: Also at Suleyman Demirel University, Isparta, Turkey

44: Also at Ege University, Izmir, Turkey

45: Also at School of Physics and Astronomy, University of Southampton, Southampton, U.K.

46: Also at INFN Sezione di Perugia; Università di Perugia, Perugia, Italy

47: Also at Utah Valley University, Orem, USA

48: Also at Institute for Nuclear Research, Moscow, Russia

49: Also at University of Belgrade, Faculty of Physics and Vinca Institute of Nuclear Sciences, Belgrade, Serbia

50: Also at Los Alamos National Laboratory, Los Alamos, USA

51: Also at Argonne National Laboratory, Argonne, USA

52: Also at Erzincan University, Erzincan, Turkey

53: Also at Kyungpook National University, Daegu, Korea 